\title{
The impact of China's WTO accession on regional economies
}

\section{Tingsong Jiang}

China was admitted to the WTO in November 2001, after making commitments far beyond those most member economies agreed to when they joined (Lardy 2002). The accession ended a 15-year long and difficult negotiation process. However, the discussion of the impact of China's accession on the domestic economy and on the world economy has only just begun.

Many studies discuss the impact of China's accession using general equilibrium models, because these models enable panoramic analysis of economy-wide effects. ${ }^{1}$ These studies share the view that, overall, China will achieve gains in economic efficiency but that agriculture, the auto industry and the banking system are vulnerable sectors.

Few studies have tried to investigate the impact of WTO accession on regional development. Yang and Huang (1997) and Jiang (2002a) use different types of representative households to approximate the regional impacts of trade liberalisation and WTO accession. Diao, Fan and Zhang (2002) and Diao et al (2002) present a general equilibrium model with partial disaggregation, that is, distinguishing nine different regions but only for the agricultural sectors. Fan and Zheng $(2000,2001)$ discuss the regional impact of trade liberalisation in their PRCGEM model following the topdown approach. However, their analysis is incomplete because it attributes the regional impact only to the difference in sectoral composition. One 
reason for this limitation is that constructing a multi-regional CGE model of the Chinese economy requires detailed regional input-output, income, consumption and trade data, all of which are often difficult to obtain.

Although difficulties exist, the regional impacts of WTO accession deserve the same attention as the sectoral impacts. China is a big country in many senses, with huge regional differences in geographic and economic terms. Regional income disparity worsened along with economic reform and growth during the 1990s. Income disparity has become so large that the central government announced its 'West Development Strategy' in 2000. Analysis of sectoral effects is a part of the investigation into the regional effects of WTO accession; therefore, a regional analysis could provide a more comprehensive picture of the impacts.

\section{Impact of WTO accession on China's regional economies}

China has made a WTO-plus commitment. It promised not only to reduce significantly tariff and non tariff barriers but also to open up sectors such as telecommunications, banking, insurance, asset management and distribution of foreign investment. It also agreed to abide by all WTO rules. Moreover, China has been forced to accept discriminatory treatment in two important rule-based areas: safeguards and antidumping (Lardy 2002).

It is difficult to accommodate all of China's commitments in one simulation. Rather, this study investigates the impact of the most obvious and simplest commitment: the required tariff cut. Even this is not as easy as it may seem. China's import tariffs are often subject to exemption and reduction under special arrangements that make the effective tariff rates significantly different from the statutory rates. For example, the average statutory tariff rate was 16.4 per cent in 2000, while tariff revenue accounted for only 4.03 per cent of the value of imports (National Bureau of Statistics of China 2001).

The database of the model represents the Chinese economy in 2000, with the average tariff rate being 16.2 per cent, which is close to the statutory tariff rate. ${ }^{2}$ The tariff rates used in this study (Table A6.2) are mainly drawn from the GTAP Database 5 with some revisions based on other studies (for example Wang 2000, lanchovichina and Martin 2001 and Anderson, Huang and Ianchovichina 2002). The WTO tariff rates are only approximately 
consistent with the actual commitments (Annex 8: Schedule CLII of Protocol on the Accession of the People's Republic of China, 2001).

\section{Closures of the model for the simulations}

Endowments of primary factors are treated as exogenous. There are slack labour variables to allow unemployment. But in the simulations described below these slack variables are set to be zero, leaving wages to adjust for full employment. ${ }^{3}$

All tax rates, including tariff rates, and technological shifters are set exogenously. Exogenous tax rates imply that government revenues will change along with changes in production, income and trade after shocks. This form of closure differs from a closure where tax rates are adjusted so as to raise a fixed amount of revenues. This particular form of closure is chosen because we want to identify the impact of WTO accession from other policy changes. The shares of transfer payments from the central government to regional governments do not change, that is, payments to each region change at the same rate.

The propensities to save are fixed, although they vary across households and regions. The difference between national savings and aggregate investment is the net capital inflow, which is equal to the trade deficit. There are two closures in the following simulation.

- No control on the trade balance. In this closure, the nominal exchange rate is fixed, the trade balance is endogenous, and foreign capital flows automatically match the balance.

- No change in the trade balance. In this closure, a floating exchange rate regime is assumed so that the change in the trade balance can be exogenously fixed at zero. ${ }^{4}$

One might think that the first closure is the more natural one, involving only tariff cuts in the simulation. However, it may still require some other policy changes to validate the closure. For example, it requires capital inflows to match the trade deficit at whatever level the model generates, implying that there is no control on foreign investment. This is clearly not the case. But as it is expected that foreign investment will increase after WTO accession (Chen 2002), and zero change seems too extreme, one would 
expect that the real situation lies somewhere between the two closures, although perhaps closer to the former.

Simulation results of the tariff cut can be found in Tables 6.1 and 6.2 and Tables A6.3 to A6.5. Table 6.1 reports the macroeconomic effects of tariff cuts under different closures, and Table 6.2 reports the impact of tariff cuts on regional output, imports and exports of aggregated commodities or sectors. The disaggregated sectoral results are reported in Tables A6.3 to A6.5.

Table 6.1 Macroeconomic effects of tariff cuts

Indications

No control on trade balance

No change in trade balance

Eastern Central Western National Eastern Central Western National

$\begin{array}{lcccccccc}\text { Real GDP(\%) } & 0.87 & -0.06 & 0.33 & 0.56 & 0.85 & -0.07 & 0.33 & 0.54 \\ \text { GDP deflator(\%) } & -1.90 & -2.03 & -2.79 & -2.07 & 3.35 & 3.04 & 2.28 & 3.11 \\ \text { CPI (\%) } & & & & & & & & \\ \quad \text { Rural households } & -2.66 & -2.50 & -3.11 & -2.70 & 2.42 & 2.51 & 1.90 & 2.35 \\ \quad \text { Urban households } & -2.56 & -2.27 & -2.77 & -2.53 & 2.55 & 2.82 & 2.33 & 2.57 \\ \quad \text { Government } & -0.67 & -1.30 & -1.33 & -0.95 & 4.72 & 3.98 & 3.97 & 4.40 \\ \quad \text { Regional average } & -2.61 & -2.40 & -2.95 & -2.62 & 2.48 & 2.64 & 2.10 & 2.45 \\ \text { Total utility (\%) } & & & & & & & & \\ \quad \text { Rural households } & 0.67 & 0.22 & 0.17 & 0.46 & 0.35 & 0.01 & 0.02 & 0.20 \\ \quad \text { Urban households } & 1.85 & 1.16 & 1.36 & 1.62 & 1.30 & 0.69 & 1.05 & 1.13 \\ \quad \text { Government } & 0.49 & -1.94 & -2.47 & -0.37 & 1.41 & -2.01 & -2.54 & 0.23 \\ \quad \text { Regional average } & 1.19 & 0.30 & 0.48 & 0.86 & 0.98 & 0.02 & 0.25 & 0.63 \\ \text { Equivalent variation (billion yuan) } & & & & & & & \\ \quad \text { Rural households } & 11.86 & 2.32 & 0.83 & 15.01 & 6.23 & 0.13 & 0.1 & 6.46 \\ \quad \text { Urban households } & 38.68 & 7.89 & 9.79 & 56.35 & 27.08 & 4.66 & 7.53 & 39.26 \\ \text { Government } & 5.57 & -6.84 & -6.40 & -7.67 & 15.97 & -7.07 & -6.58 & 2.32 \\ \quad \text { Regional sum } & 56.11 & 3.36 & 4.22 & 63.69 & 49.28 & -2.28 & 1.04 & 48.04 \\ \text { Savings (nominal, per cent) } & & & & & & & \\ \quad \text { Rural households } & -1.47 & -2.03 & -2.64 & -1.76 & 3.58 & 2.97 & 2.28 & 3.26 \\ \quad \text { Urban households } & -0.14 & -1.07 & -1.04 & -0.48 & 5.29 & 4.25 & 4.29 & 4.91 \\ \quad \text { Government } & -3.45 & -3.26 & -3.78 & -3.44 & 1.63 & 1.85 & 1.32 & 1.64 \\ \quad \text { Regional average } & -1.02 & -1.66 & -1.41 & -1.22 & 4.24 & 3.47 & 3.84 & 4.01 \\ \text { Nominal exchange rate } & & & & - & & & & 5.45 \\ \text { Change in trade balance (billion yuan) } & & -42.55 & & & & - \\ \text { Terms of trade (\%) } & & & & -0.46 & & & & -0.47\end{array}$

Source: Author's CERD simulation. 
Simulating with no control on the trade balance

For this simulation it can be seen from Table 6.1 that China has a net gain from WTO accession. By cutting the tariff rates as listed in Table A6.2, China's real GDP would increase by 0.56 per cent, utility by 0.86 per cent, and the equivalent variation, a welfare indicator, would reach 63.69 billion yuan. Higher welfare comes from higher real incomes, thus higher real consumption and saving. But the tariff cut has an adverse impact on the trade balance and the terms of trade. China's trade balance declines by 42.55 billion yuan because imports increase more than exports and the terms of trade decreases by 0.46 per cent. This is because the tariff cut does not affect the border price (CIF). However, Chinese exports become cheaper because tariff cuts help to lower production costs.

It can be seen from Tables 6.2 and A6.3 that the impact will not be evenly distributed. The motor vehicle and other transport equipment sector is the biggest loser, with output declining by 16.37 per cent. It is followed by the food and tobacco processing, machinery and chemicals sectors, where output would decline by more than 3 per cent. The impact on the agricultural sectors would not be as severe as some studies have suggested. Crop output declines by 1.2 per cent and total agricultural output falls by less than 0.1 per cent. These results are in line with the results in lanchovichina and Martin (2001). The smaller decline in agricultural output may be partly due to the smaller extent of the tariff cuts. It may be also partly attributed to the aggregation of agricultural sectors. If the crop sector could be disaggregated to individual crops, it may be that the output of some crops would fall significantly.

Table A6.3 shows that the sector with the highest growth would be apparels, with a more than 14 per cent increase in output; followed by electronics (9.93 per cent), textiles (6.34 per cent) and instruments and cultural and office machinery (3.95 per cent).

The changes in imports and exports are consistent with the changes in output. Imports of other agricultural products, food and tobacco processing, motor vehicles and other transportation equipment are more than doubled after WTO accession and crops imports increase by 88 per cent. ${ }^{5}$ Increases in exports of apparel, textiles and electronics are of a smaller magnitude. In general, these changes reflect the comparative advantage and disadvantage of Chinese industries. 
Table 6.2 Impact of tariff cuts on regional output, exports and imports

\begin{tabular}{|c|c|c|c|c|c|c|c|c|}
\hline \multirow[t]{2}{*}{ Sector ${ }^{\mathrm{a}}$} & \multicolumn{4}{|c|}{ No control on trade balance } & \multicolumn{4}{|c|}{ No change in trade balance } \\
\hline & Eastern & Central & Western & National & Eastern & Central & Western & Nationa \\
\hline \multicolumn{9}{|l|}{ Output } \\
\hline agri & - & -0.13 & -0.08 & -0.06 & -0.06 & -0.17 & -0.12 & -0.10 \\
\hline mine & -1.73 & 1.24 & 2.60 & -0.03 & -1.88 & 1.40 & 2.76 & -0.02 \\
\hline fprc & -4.20 & -1.71 & -5.35 & -3.59 & -4.33 & -1.80 & -5.53 & -3.71 \\
\hline lind & 7.68 & 0.04 & 2.79 & 5.86 & 7.77 & 0.20 & 3.37 & 6.00 \\
\hline chem & -2.02 & -0.96 & -0.75 & -1.63 & -2.04 & -0.90 & -0.69 & -1.63 \\
\hline motr & -17.88 & -14.87 & -11.54 & -16.37 & -18.05 & -14.58 & -11.10 & -16.36 \\
\hline mche & -3.86 & -0.95 & 0.29 & -2.76 & -3.92 & -0.75 & 0.49 & -2.73 \\
\hline elen & 5.72 & -0.85 & 3.10 & 4.98 & 5.75 & -0.76 & 3.38 & 5.03 \\
\hline cnst & 0.46 & 1.09 & 1.18 & 0.72 & 0.43 & 1.11 & 1.20 & 0.72 \\
\hline svce & 0.82 & -0.13 & 0.15 & 0.52 & 0.87 & -0.22 & 0.07 & 0.50 \\
\hline \multicolumn{9}{|l|}{ Exports } \\
\hline agri & 17.19 & 15.07 & 17.41 & 16.56 & 20.21 & 17.83 & 19.90 & 19.44 \\
\hline mine & 1.73 & 8.15 & 9.24 & 4.74 & 1.83 & 8.78 & 9.76 & 5.05 \\
\hline fprc & 9.58 & 9.67 & 6.92 & 9.19 & 10.95 & 11.09 & 7.85 & 10.50 \\
\hline lind & 21.70 & 8.87 & 19.21 & 20.03 & 22.37 & 9.93 & 21.78 & 20.82 \\
\hline chem & 4.89 & 5.97 & 6.65 & 5.15 & 5.16 & 6.49 & 7.20 & 5.48 \\
\hline motr & -10.31 & -5.47 & 0.80 & -7.86 & -10.37 & -4.81 & 1.59 & -7.70 \\
\hline mche & 1.20 & 5.82 & 7.47 & 2.60 & 1.32 & 6.45 & 8.01 & 2.84 \\
\hline elen & 14.94 & 5.68 & 12.77 & 14.39 & 15.23 & 6.16 & 13.50 & 14.72 \\
\hline cnst & 2.53 & 5.26 & 5.37 & 3.04 & 2.58 & 5.54 & 5.59 & 3.11 \\
\hline svce & 2.83 & 4.06 & 4.90 & 3.32 & 2.86 & 4.29 & 5.11 & 3.41 \\
\hline \multicolumn{9}{|l|}{ Imports } \\
\hline agri & 47.47 & 39.20 & 63.54 & 47.97 & 44.21 & 36.35 & 60.84 & 44.82 \\
\hline mine & -4.45 & -4.95 & -4.38 & -4.53 & -4.72 & -5.14 & -4.53 & -4.77 \\
\hline fprc & 155.48 & 173.44 & 158.17 & 158.30 & 151.10 & 169.02 & 154.93 & 154.15 \\
\hline lind & 22.54 & 11.02 & 5.03 & 19.02 & 21.96 & 9.85 & 4.02 & 18.31 \\
\hline chem & 22.45 & 19.27 & 20.82 & 21.75 & 22.17 & 18.85 & 20.41 & 21.44 \\
\hline motr & 110.89 & 107.67 & 87.98 & 106.08 & 110.06 & 106.43 & 87.27 & 105.17 \\
\hline mche & 12.55 & 13.41 & 12.59 & 12.69 & 12.30 & 13.05 & 12.33 & 12.42 \\
\hline elen & 6.63 & 3.88 & 4.39 & 5.93 & 6.31 & 3.40 & 4.03 & 5.58 \\
\hline cnst & -2.19 & -4.06 & -4.03 & -3.17 & -2.31 & -4.34 & -4.25 & -3.36 \\
\hline Svce & -2.18 & -4.93 & -5.14 & -3.44 & -2.31 & -5.37 & -5.52 & -3.70 \\
\hline
\end{tabular}

Note: ${ }^{a}$ sector code: agri: agriculture; mine: mining; fprc: food processing; lind: light industry; chem: chemicals; motr: motor vehicle and other transportation equipment; mche: machinery and equipment; elen: electronics and electric equipment; cnst: construction; svce: services. Source: Author's CERD simulation. 
The simulation results also show that the eastern coastal region gains more from the WTO accession than the inland regions. The order of increase in total utility and equivalent variation is: eastern, western and central. This result suggests that regional income disparity would worsen after the accession, although all regions may gain. The eastern region gains most of the benefit from the expanding sectors. For example, apparel output in the eastern region increases by over 17 per cent, while this sector's outputs in the central and western regions increase by only 0.3 per cent and 1.5 per cent, respectively (Table A6.3). Similarly, textile output in the eastern region increases the most, while it falls in the central region.

The results show that the output level in the central region may decline after WTO accession, as indicated by the negative change in real GDP. This result seems surprising because it is generally perceived that the western region is the least developed region and thus should be the most adversely affected. However, the result may be justified in the following way. First, the western region has the cheapest labour, which helps in the development of labour-intensive sectors. Second, the western region has relatively abundant natural resource endowments, which leads to its comparative advantage in resource-intensive products. Finally, the industrial base in the western region may not be as poor as people think. The Chinese government has made huge investments in the so-called 'third line' program which brought about development in some sectors.

The results of this simulation show that WTO accession would worsen rural-urban income inequality in all regions. That rural households experience a smaller increase in utility and welfare is understandable because rural households receive part of their income from agricultural sectors, which decline in all regions following WTO accession.

\section{Simulating with no change in the trade balance}

This closure would have a similar impact on regional economies, despite some differences in the macroeconomic dimensions. The fixed exchange rate in the previous closure brings about domestic deflation, while the fixed trade balance with a floating exchange rate leads to depreciation of the RMB by 5.5 per cent-which in turn causes domestic inflation of 2.45 per 
cent. In both cases, the real exchange rate increases; but in the present form of closure the increase is slightly greater (3.0 per cent versus 2.6 per cent) (Table 6.1).

This form of closure gives a smaller welfare gain than the previous one because it restrains the gains from trade by fixing the trade balance. Moreover, it causes an even wider welfare gap. In the previous closure, the eastern region's share in the total equivalent variation is 88 per cent. In the current closure, all the gains are absorbed by the eastern region while the inland regions have a net loss. The gap between rural and urban households is also likely to widen. Urban households' share in the total household welfare gain increases from 79 per cent to 86 per cent (Table 6.1).

Why does WTO accession worsen regional disparity?

To some, it may be surprising, even unacceptable, that trade liberalisation should lead to worsening regional disparity in China. However, further analysis may reveal that it is a necessary result.

First, it is a natural extension of the historical trend. As shown in Figure 6.1, the income gap between China's coastal and inland regions has been widening since the economic reforms in 1978. Of the three regions defined here, the richest eastern coastal region has experienced the fastest economic growth over the past two decades. The average per capita GDP in the eastern region has increased sevenfold, while per capita GDP in the poorest western region has increased by less than five times. Consequently, the gap in per capita GDP between the eastern and western regions increased from 380.6 yuan in 1978 to 3354.9 yuan in 2000 in real terms. This phenomenon has been well documented by many authors (for example Wu 1999, Sun 2000, Sun and Parikh 2001 and Jiang 2002).

Second, the regional economies in China are segregated, and this segregation is embodied in both factor and commodity markets. The labour factor market segregation takes legal form in the household registration system. The general equilibrium CERD model captures this feature. Because labour is not freely mobile, wage differentials between regions increase after the WTO accession, which leads to worsening regional income disparity. The model assumes perfect mobility of capital, which may not be the case in 
Figure 6.1 Per capita GDP by region, 1978-2000
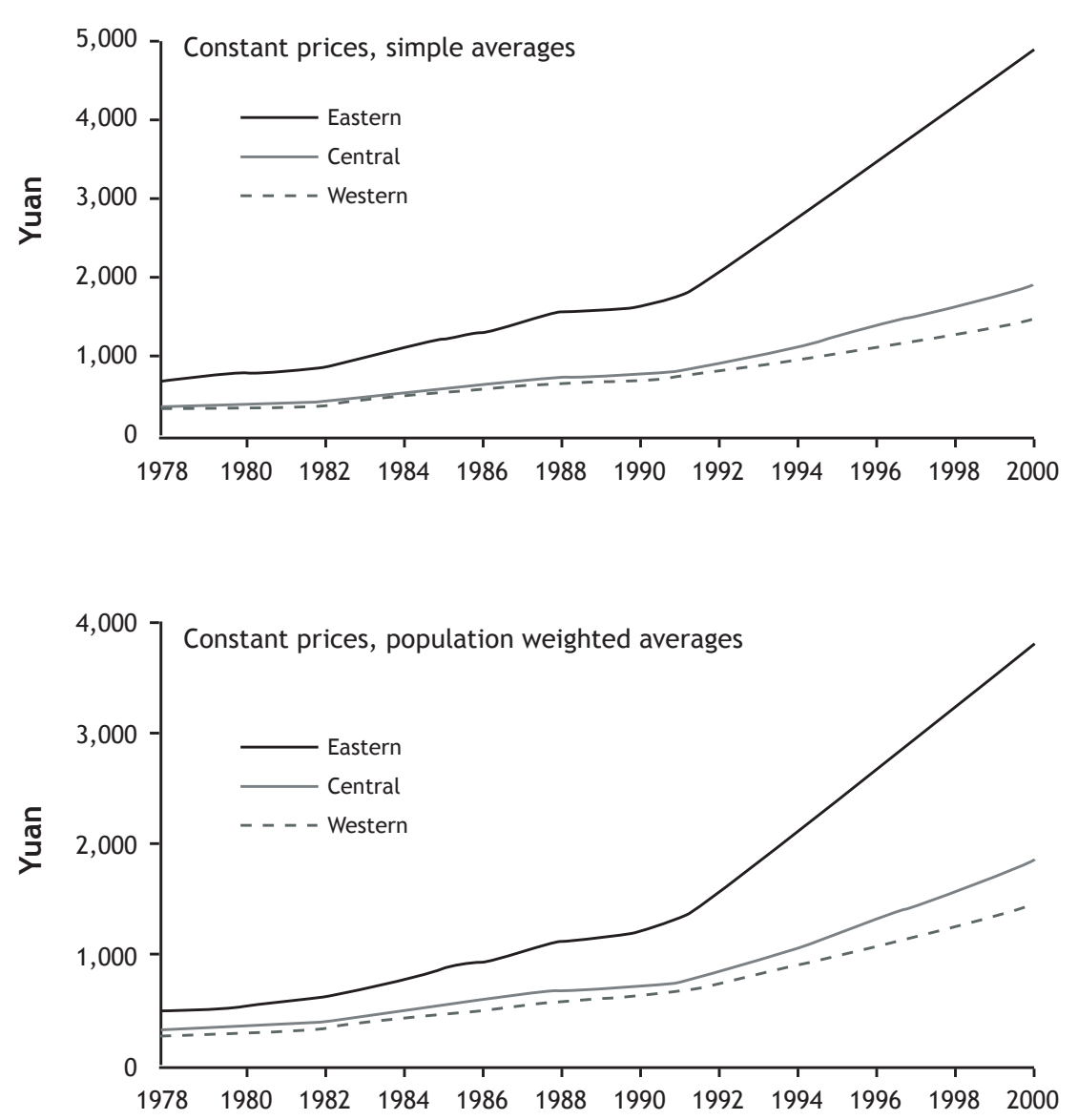

Source: Author's construction based on data from National Bureau of Statistics of China, 2001. Statistical Yearbook of China, China Statistics Press, Beijing. 
reality. However, it captures some effects on regional disparity. Although the rate of return to capital is the same across regions, the coastal region still gains more than the inland regions as it owns a higher proportion of the capital stock.

It is commonly recognised that regional protectionism prevails in China, which prevents the integration of commodity markets. Because commodities are not to be freely traded between regions within China even after the WTO accession, factor price equalisation cannot be achieved. The CERD model captures this effect through distinguishing regional commodity markets and assuming imperfect substitution between the same commodity sourced from different regions.

Third, the technological levels are different in the three regions, which violates another underlying assumption of the factor price equalisation hypothesis. The differences in technology are embodied in the database of the CERD model. The coastal region has more advanced technology than the inland regions, and, thus, accrues higher welfare gains after the WTO accession.

It is argued that simulating only tariff cuts tends to underestimate the impact of trade liberalisation (Kehoe 2002). Considering the high probability of higher technological progress following the WTO accession, the regional income disparity may be even worse than presented above. This is because the coastal region could well experience faster technological progress than the inland regions. This is evident as the foreign direct investment has been and will be concentrated in the coastal area.

Fourth, because the economy is regionally segregated, the difference in regional economic structure also results in worsening income disparity. As shown above, the coastal region has a higher proportion of most of the expanding sectors following the WTO accession, while the inland regions have a higher proportion of contracting sectors.

\section{Policy simulations}

From the above discussion, some policy recommendations may be raised. This section presents the simulation results of three important policies: additional protection to the agricultural sector; domestic market liberalisation; and government transfer payments to inland regions. 


\section{Additional protection to agricultural sectors}

Many studies of China's WTO accession have projected that agriculture will be one of the hardest hit sectors. The simulations reported above confirm that agriculture will be adversely affected by the WTO accession, leading to a worsening of regional income disparities as the inland regions have a higher proportion of agricultural activities in their economies. Therefore, the Chinese government (and academic circles) have been worrying about this issue and adopted some measures to anticipate the detrimental outcomes. ${ }^{6,7}$ These measures provide additional protection to agriculture.

To capture this additional protection to agriculture, the simulations presented in the previous section are revised with the tariff cuts in the agricultural sectors being only half of the level in the previous section, ceteris paribus. The simulation results are reported in Tables 6.3 and 6.4. Several points are evident from these results.

First, such policy actions do provide some cushioning effects to agricultural sectors. The increase in agricultural imports is smaller, falling from 47.3 per cent to 15.2 per cent with the closure of no control on the trade balance, and from 44.2 per cent to 13.1 per cent with the closure of no change in the trade balance. Consequently, agricultural output declines by only 0.03 and 0.07 per cent, respectively. Second, because the central region is the major agricultural production area, this protection helps the central region achieve higher welfare (up from 3.36 billion yuan to 4.12 billion yuan) with the closure of no control on the trade balance, or lower welfare loss (from -2.28 billion yuan to -0.50 billion yuan). Third, it also helps to ease the worsening rural-urban inequality. Rural households have a higher growth in utility than in the previous scenario while urban households have a lower growth. Fourth, the negative impact on the trade balance and the terms of trade is now smaller. Finally, however, the total welfare gain is smaller than for the full tariff cuts set by the WTO agreement.

\section{Domestic market reform}

It is often argued that China's domestic economy is not well integrated and heavy regional protection exists. One prominent example is the restriction on the movement of people. Also, in many regions the use of 
Table 6.3 Macroeconomic effects of WTO tariff cuts with agricultural protection

Indicators

No control on trade balance

Eastern Central Western National
No change in trade balance

Eastern Central Western National

$\begin{array}{lrrrrrrrr}\text { Real GDP (\%) } & 0.77 & -0.10 & 0.28 & 0.48 & 0.75 & -0.10 & 0.27 & 0.46 \\ \text { GDP deflator (\%) } & -1.74 & -1.82 & -2.53 & -1.89 & 2.50 & 2.27 & 1.56 & 2.29 \\ \text { CPI (\%) } & & & & & & & & \\ \quad \text { Rural households } & -2.24 & -2.12 & -2.62 & -2.28 & 1.86 & 1.92 & 1.42 & 1.79 \\ \quad \text { Urban households } & -2.23 & -2.05 & -2.51 & -2.24 & 1.89 & 2.05 & 1.60 & 1.88 \\ \quad \text { Government } & -0.77 & -1.39 & -1.44 & -1.04 & 3.57 & 2.86 & 2.83 & 3.27 \\ \quad \text { Regional average } & -2.24 & -2.09 & -2.57 & -2.26 & 1.87 & 1.98 & 1.50 & 1.83 \\ \text { Total utility (\%) } & & & & & & & & \\ \quad \text { Rural households } & 0.91 & 0.44 & 0.49 & 0.70 & 0.64 & 0.27 & 0.36 & 0.48 \\ \quad \text { Urban households } & 1.58 & 0.91 & 1.09 & 1.35 & 1.14 & 0.53 & 0.84 & 0.96 \\ \quad \text { Government } & 0.24 & -1.92 & -2.42 & -0.53 & 0.99 & -1.97 & -2.48 & -0.04 \\ \quad \text { Regional average } & 1.10 & 0.33 & 0.44 & 0.81 & 0.93 & 0.10 & 0.25 & 0.62\end{array}$

Equivalent variation (billion yuan)

$\begin{array}{lrrrrrrrr}\text { Rural households } & 16.02 & 4.68 & 2.34 & 23.04 & 11.34 & 2.85 & 1.71 & 15.89 \\ \text { Urban households } & 33.05 & 6.20 & 7.84 & 47.08 & 23.72 & 3.60 & 6.03 & 33.36 \\ \text { Government } & 2.67 & -6.76 & -6.28 & -10.36 & 11.19 & -6.95 & -6.44 & -2.19 \\ \text { Regional sum } & 51.75 & 4.12 & 3.89 & 59.76 & 46.25 & -0.50 & 1.31 & 47.06\end{array}$

Savings (nominal, per cent)

Rural households $\quad-1.03$

$\begin{array}{lllllll}-1.59 & -1.98 & -1.30 & 3.05 & 2.44 & 2.00 & 2.75\end{array}$

Urban households

$-0.30$

$-1.19$

$-1.19$

$-0.64$

4.07

3.08

3.10

3.70

Government

$-3.59$

$-3.32$

$-3.84$

$-3.58$

0.50

0.80

0.27

0.51

Regional average

$-0.97$

$-1.38-1.14$

3.26

2.67

2.84

3.07

4.39

Change in trade balance (billion yuan)

$-34.42$

$-0.43$

Terms of trade (\%)

$-0.42$

Source: Author's CERD simulation. 
Table 6.4 Impact on output, exports and imports of WTO tariff cuts with agricultural protection

\begin{tabular}{|c|c|c|c|c|c|c|c|c|}
\hline \multirow[t]{2}{*}{ Sector ${ }^{\mathrm{a}}$} & \multicolumn{4}{|c|}{ No control on trade balance } & \multicolumn{4}{|c|}{ No change in trade balance } \\
\hline & Eastern & Central & Western & National & Eastern & Central & Western & National \\
\hline \multicolumn{9}{|l|}{ Output } \\
\hline Agri & - & -0.06 & -0.05 & -0.03 & -0.05 & -0.09 & -0.07 & -0.07 \\
\hline Mine & -1.49 & 1.24 & 2.78 & 0.12 & -1.62 & 1.37 & 2.91 & 0.12 \\
\hline Fprc & -5.33 & -1.80 & -5.41 & -4.24 & -5.42 & -1.87 & -5.55 & -4.33 \\
\hline Lind & 7.35 & -0.22 & 1.34 & 5.47 & 7.43 & -0.08 & 1.81 & 5.59 \\
\hline Chem. & -1.92 & -0.95 & -0.75 & -1.57 & -1.94 & -0.91 & -0.69 & -1.56 \\
\hline Motr & -17.62 & -14.71 & -11.12 & -16.10 & -17.76 & -14.48 & -10.76 & -16.09 \\
\hline Mche & -3.61 & -0.90 & 0.49 & -2.56 & -3.66 & -0.73 & 0.65 & -2.54 \\
\hline Elen & 6.43 & -0.79 & 3.49 & 5.61 & 6.44 & -0.71 & 3.73 & 5.65 \\
\hline Cnst & 0.44 & 1.04 & 1.16 & 0.69 & 0.42 & 1.06 & 1.17 & 0.69 \\
\hline Svce & 0.75 & -0.13 & 0.14 & 0.48 & 0.80 & -0.21 & 0.08 & 0.48 \\
\hline \multicolumn{9}{|l|}{ Exports } \\
\hline Agri & 11.45 & 10.88 & 12.57 & 11.39 & 13.88 & 13.11 & 14.6 & 13.72 \\
\hline Mine & 2.63 & 8.55 & 10.06 & 5.48 & 2.69 & 9.05 & 10.48 & 5.73 \\
\hline Fprc & 5.25 & 7.75 & 5.08 & 5.86 & 6.38 & 8.92 & 5.86 & 6.95 \\
\hline Lind & 20.99 & 8.12 & 14.57 & 19.14 & 21.53 & 8.98 & 16.6 & 19.79 \\
\hline Chem. & 5.25 & 6.29 & 6.89 & 5.50 & 5.47 & 6.71 & 7.33 & 5.76 \\
\hline Motr & -9.64 & -4.95 & 1.71 & -7.17 & -9.70 & -4.42 & 2.36 & -7.04 \\
\hline Mche & 1.92 & 6.32 & 8.21 & 3.29 & 2.01 & 6.83 & 8.65 & 3.48 \\
\hline Elen & 16.33 & 6.14 & 13.8 & 15.72 & 16.55 & 6.53 & 14.39 & 15.97 \\
\hline Cnst & 2.85 & 5.50 & 5.67 & 3.35 & 2.88 & 5.73 & 5.85 & 3.41 \\
\hline Svce & 3.05 & 4.29 & 5.17 & 3.54 & 3.08 & 4.47 & 5.33 & 3.62 \\
\hline \multicolumn{9}{|l|}{ Imports } \\
\hline Agri & 15.25 & 11.36 & 20.17 & 15.21 & 13.08 & 9.42 & 18.43 & 13.11 \\
\hline Mine & -4.86 & -5.37 & -4.76 & -4.93 & -5.07 & -5.52 & -4.88 & -5.12 \\
\hline Fprc & 161.79 & 177.93 & 162.41 & 163.94 & 158.03 & 174.18 & 159.65 & 160.40 \\
\hline Lind & 22.54 & 11.45 & 5.62 & 19.15 & 22.06 & 10.49 & 4.78 & 18.56 \\
\hline Chem. & 22.27 & 19.05 & 20.63 & 21.57 & 22.05 & 18.71 & 20.30 & 21.32 \\
\hline Motr & 109.31 & 106.26 & 86.76 & 104.61 & 108.68 & 105.28 & 86.20 & 103.90 \\
\hline Mche & 12.11 & 12.86 & 12.06 & 12.22 & 11.92 & 12.58 & 11.86 & 12.01 \\
\hline Elen & 6.33 & 3.31 & 3.88 & 5.57 & 6.07 & 2.93 & 3.60 & 5.28 \\
\hline Cnst & -2.64 & -4.44 & -4.45 & -3.60 & -2.72 & -4.66 & -4.62 & -3.73 \\
\hline Svce & -2.54 & -5.21 & -5.45 & -3.77 & -2.64 & -5.56 & -5.76 & -3.98 \\
\hline
\end{tabular}

Note: ${ }^{a}$ sector code: agri: agriculture; mine: mining; fprc: food processing; lind: light industry; chem: chemicals; motr: motor vehicle and other transportation equipment; mche: machinery and equipment; elen: electronics and electric equipment; cnst: construction; svce: services.

Source: Author's CERD simulation. 
land is controlled by governments. It is also frequently reported that local governments erect barriers to bar products from other regions. However, there are no complete and accurate data to enable the calculation of tarifflike protection in Chinese regions. For this reason, a hypothetical scenario is constructed to investigate this issue. Specifically, a set of elasticities are chosen to imitate domestic market reforms.

Elasticities of transformation or substitution usually describe the difference between two goods or factors. However, they may also reflect how easily one good or factor can be transformed or substituted into another. For example, a higher elasticity of transformation between rural and urban labour also describes the higher mobility of labour from rural to urban areas. In this sense, the domestic market reform can be represented by increases in selected elasticities. These elasticities include the elasticity of transformation between agricultural, non agricultural and migrant labour (from 0.1 to 1); the elasticity of transformation between different land uses (from 0.05 to 0.5 ); the elasticity of substitution between commodities produced locally and in other regions (Table 6.5); and the elasticity of substitution between different types of labour (from 4 to 14).

Table 6.5 Elasticities of substitution between local and other products

\begin{tabular}{lccllllllllll} 
Comm & Original & New & \multicolumn{2}{l}{ Comm Original New } & \multicolumn{3}{c}{ Comm Original New } & \multicolumn{2}{c}{ Comm Original New } \\
crop & 4.4 & 10.4 & aprl & 8.8 & 10.4 & eltn & 5.6 & 10.4 & trad & 3.8 & 10.4 \\
Frst & 5.6 & 10.4 & furn & 5.6 & 10.4 & inst & 5.6 & 10.4 & cate & 3.8 & 10.4 \\
Live & 4.6 & 10.4 & papr & 3.6 & 10.4 & main & 5.6 & 10.4 & past & 3.8 & 10.4 \\
Fish & 5.6 & 10.4 & ptpc & 3.8 & 10.4 & omnp & 5.6 & 10.4 & fina & 3.8 & 10.4 \\
Otha & 5.6 & 10.4 & chem & 3.8 & 10.4 & scrp & 5.6 & 10.4 & rest & 3.8 & 10.4 \\
coal & 5.6 & 10.4 & nmmp & 5.6 & 10.4 & powr & 5.6 & 10.4 & sser & 3.8 & 10.4 \\
Petr & 5.6 & 10.4 & mtsp & 5.6 & 10.4 & gasp & 5.6 & 10.4 & heth & 3.8 & 10.4 \\
mtom & 5.6 & 10.4 & mtlp & 5.6 & 10.4 & watr & 5.6 & 10.4 & educ & 3.8 & 10.4 \\
nmtm & 5.6 & 10.4 & mach & 5.6 & 10.4 & cons & 3.8 & 10.4 & scir & 3.8 & 10.4 \\
Fdtp & 4.9 & 10.4 & treq & 10.4 & 10.4 & tran & 3.8 & 10.4 & teks & 3.8 & 10.4 \\
Txtl & 4.4 & 10.4 & eltc & 5.6 & 10.4 & ptlc & 3.8 & 10.4 & padm & 3.8 & 10.4 \\
& & & & & & & & & & & \\
\hline
\end{tabular}

Note: See sector classification abbreviations at Table A6.1.

Source: Author's construction. 
With these new values, the inter-regional factor and commodity flows become more intensive. For example, in the closure of no control on the trade balance, the price of migrant labour declines by 0.18 per cent when the elasticities are higher while it increases by 0.18 per cent with the former elasticities. Consequently, migrant labour supply increases by 1.47 per cent (previously the increase was only 0.25 per cent). The migrant labour supply increases in all regions, with the western region increasing the most (1.98 per cent versus 0.31 per cent), followed by the central region ( 1.68 per cent versus 0.26 per cent) and the eastern region $(0.83$ per cent versus 0.20 per cent). Similar changes can be observed in respect of inter-regional trade of commodities. These results are understandable and do not need more explanation. It is more important to look at the welfare implications. The summary results are reported in Tables 6.6 and 6.7 while the detailed sectoral results are in Tables A6.9-A6.11.

It can be seen from the table that with the new values of parameters, WTO accession leads to higher welfare gains. The total welfare gain increases from 63.69 billion yuan in the closure with no control on the trade balance and 48.04 billion yuan in the closure with no change of the trade balance to 69.04 and 56.79 billion yuan, respectively. Although there is still a worsening of the regional income disparity, its magnitude is smaller when factor and commodities are allowed to move more freely. Every region has a higher welfare gain than before, and the shares of inland regions in the total gain increase from 12 per cent to 14 per cent. The increment in the rural households' utility and welfare after the WTO accession is higher with the revised elasticities than with former ones, while the increment in the urban households' utility and welfare is now smaller-implying a smaller degree of the rural-urban inequality.

\section{Transfer payments}

A counterfactual transfer payment program is also simulated using the CERD model. The program simply involves a 10 per cent increase in the central government's transfer payments to the central and western regions. The results are reported in Tables 6.8, 6.9 and A6.12-A6.14.

The simulation results do not give a clear justification for such a program. Although the regional and rural-urban inequality improves slightly with 
Table 6.6 Macroeconomic effects of WTO tariff cuts: higher elasticities

Indicators

No control on trade balance No change in trade balance

Eastern Central Western National Eastern Central Western National

$\begin{array}{lcccccccc}\text { Real GDP (\%) } & 0.93 & -0.12 & 0.29 & 0.57 & 0.91 & -0.12 & 0.28 & 0.56 \\ \text { GDP deflator (\%) } & -1.88 & -1.76 & -2.47 & -1.94 & 2.52 & 2.53 & 1.82 & 2.41 \\ \text { CPI (\%) } & & & & & & & & \\ \quad \text { Rural households } & -2.37 & -2.14 & -2.70 & -2.36 & 1.93 & 2.13 & 1.56 & 1.92 \\ \quad \text { Urban households } & -2.34 & -2.05 & -2.53 & -2.31 & 1.97 & 2.25 & 1.77 & 2.00 \\ \quad \text { Government } & -0.84 & -1.39 & -1.39 & -1.07 & 3.63 & 3.01 & 3.03 & 3.37 \\ \quad \text { Regional average } & -2.35 & -2.10 & -2.62 & -2.34 & 1.95 & 2.18 & 1.66 & 1.96 \\ \text { Total utility (\%) } & & & & & & & & \\ \quad \text { Rural households } & 1.02 & 0.46 & 0.51 & 0.77 & 0.81 & 0.33 & 0.43 & 1.92 \\ \quad \text { Urban households } & 1.65 & 0.93 & 1.16 & 1.41 & 1.15 & 0.50 & 0.87 & 2.00 \\ \quad \text { Government } & 0.62 & -1.80 & -2.33 & -0.24 & 1.41 & -1.83 & -2.37 & 3.37 \\ \quad \text { Regional average } & 1.24 & 0.36 & 0.50 & 0.91 & 1.07 & 0.13 & 0.31 & 0.72 \\ \text { Equivalent variation (billion yuan) } & & & & & & & \\ \quad \text { Rural households } & 17.97 & 4.93 & 2.41 & 25.32 & 14.22 & 3.54 & 2.05 & 19.81 \\ \quad \text { Urban households } & 34.42 & 6.34 & 8.35 & 49.11 & 23.94 & 3.37 & 6.23 & 33.54 \\ \quad \text { Government } & 7.03 & -6.36 & -6.05 & -5.39 & 16.02 & -6.44 & -6.15 & 3.43 \\ \quad \text { Regional sum } & 59.42 & 4.92 & 4.71 & 69.04 & 54.18 & 0.47 & 2.13 & 56.79 \\ \text { Savings (nominal, \%) } & & & & & & & & \\ \quad \text { Rural households } & -1.00 & -1.58 & -2.02 & -1.29 & 3.32 & 2.69 & 2.22 & 3.01 \\ \quad \text { Urban households } & -0.30 & -1.17 & -1.12 & -0.62 & 4.20 & 3.25 & 3.32 & 3.85 \\ \quad \text { Government } & -3.36 & -3.20 & -3.70 & -3.36 & 0.89 & 1.09 & 0.57 & 0.90 \\ \quad \text { Regional average } & -0.94 & -1.45 & -1.33 & -1.11 & 3.47 & 2.89 & 3.06 & 3.28 \\ \text { Nominal exchange rate } & & & & - & & & & 4.56 \\ \text { Change in trade balance (billion yuan) } & & -36.74 & & & & - \\ \text { Terms of trade (\%) } & & & & -0.47 & & & & -0.48\end{array}$

Source: Author's CERD simulation. 
Table 6.7 Impact on output, exports and imports of WTO tariff cuts: higher elasticities

\begin{tabular}{|c|c|c|c|c|c|c|c|c|}
\hline \multirow[t]{2}{*}{ Sector ${ }^{a}$} & \multicolumn{4}{|c|}{ No control on trade balance } & \multicolumn{4}{|c|}{ No change in trade balance } \\
\hline & Eastern & Central & Western & National & Eastern & Central & Western & National \\
\hline \multicolumn{9}{|l|}{ Output } \\
\hline agri & -0.83 & -0.47 & -0.46 & -0.64 & -1.00 & -0.58 & -0.53 & -0.77 \\
\hline mine & -1.81 & 1.69 & 3.15 & 0.18 & -1.93 & 1.90 & 3.32 & 0.22 \\
\hline fprc & -4.84 & -2.05 & -5.53 & -4.07 & -5.05 & -2.18 & -5.70 & -4.25 \\
\hline lind & 8.18 & -0.71 & 1.39 & 5.99 & 8.29 & -0.61 & 1.72 & 6.12 \\
\hline chem & -1.73 & -1.01 & -0.79 & -1.46 & -1.73 & -0.93 & -0.72 & -1.43 \\
\hline motr & -17.31 & -14.64 & -11.80 & -15.98 & -17.37 & -14.33 & -11.39 & -15.91 \\
\hline mche & -3.60 & -0.58 & 0.77 & -2.45 & -3.60 & -0.33 & 0.99 & -2.37 \\
\hline elen & 7.06 & -1.48 & 2.08 & 5.97 & 7.25 & -1.34 & 2.38 & 6.17 \\
\hline cnst & 0.56 & 1.22 & 1.32 & 0.83 & 0.55 & 1.26 & 1.35 & 0.84 \\
\hline svce & 0.90 & 0.04 & 0.28 & 0.63 & 0.96 & -0.01 & 0.23 & 0.65 \\
\hline \multicolumn{9}{|l|}{ Exports } \\
\hline agri & 10.23 & 10.45 & 12.59 & 10.54 & 11.64 & 11.80 & 13.87 & 11.92 \\
\hline mine & 2.02 & 9.19 & 10.30 & 5.37 & 2.18 & 9.91 & 10.88 & 5.75 \\
\hline fprc & 6.54 & 7.53 & 5.16 & 6.58 & 7.21 & 8.31 & 5.70 & 7.26 \\
\hline lind & 22.39 & 7.29 & 14.79 & 20.22 & 23.00 & 8.05 & 16.38 & 20.89 \\
\hline chem & 5.62 & 6.15 & 6.54 & 5.75 & 5.93 & 6.69 & 7.04 & 6.11 \\
\hline motr & -9.20 & -4.91 & 0.67 & -7.03 & -9.10 & -4.19 & 1.45 & -6.74 \\
\hline mche & 2.06 & 6.66 & 8.32 & 3.45 & 2.30 & 7.38 & 8.92 & 3.80 \\
\hline elen & 17.28 & 4.77 & 11.16 & 16.33 & 17.83 & 5.31 & 11.90 & 16.90 \\
\hline cnst & 3.08 & 5.68 & 5.71 & 3.55 & 3.21 & 6.01 & 5.96 & 3.70 \\
\hline svce & 3.29 & 4.45 & 5.16 & 3.74 & 3.40 & 4.72 & 5.39 & 3.89 \\
\hline \multicolumn{9}{|l|}{ Imports } \\
\hline agri & 53.53 & 43.24 & 68.15 & 53.58 & 51.52 & 41.57 & 66.50 & 51.65 \\
\hline mine & -4.90 & -4.81 & -4.28 & -4.80 & -5.18 & -4.97 & -4.44 & -5.05 \\
\hline fprc & 160.52 & 177.43 & 161.94 & 162.94 & 157.50 & 174.43 & 159.66 & 160.08 \\
\hline lind & 22.77 & 11.32 & 5.72 & 19.31 & 22.28 & 10.39 & 4.93 & 18.73 \\
\hline chem & 22.43 & 18.90 & 20.56 & 21.65 & 22.18 & 18.50 & 20.18 & 21.36 \\
\hline motr & 109.13 & 106.67 & 87.49 & 104.73 & 108.18 & 105.39 & 86.72 & 103.73 \\
\hline mche & 12.21 & 12.97 & 12.26 & 12.33 & 11.93 & 12.59 & 11.97 & 12.04 \\
\hline elen & 6.52 & 3.53 & 3.79 & 5.73 & 6.21 & 3.05 & 3.42 & 5.38 \\
\hline cnst & -2.64 & -4.36 & -4.26 & -3.52 & -2.81 & -4.66 & -4.50 & -3.74 \\
\hline Svce & -2.48 & -5.12 & -5.22 & -3.68 & -2.64 & -5.52 & -5.57 & -3.94 \\
\hline
\end{tabular}

Note: ${ }^{a}$ sector code: agri: agriculture; mine: mining; fprc: food processing; lind: light industry; chem: chemicals; motr: motor vehicle and other transportation equipment; mche: machinery and equipment; elen: electronics and electric equipment; cnst: construction; svce: services.

Source: Author's CERD simulation. 
Table 6.8 Macroeconomic effects of WTO tariff cuts: with transfer payments

Indicators

\begin{tabular}{lcccccccc} 
Real GDP (\%) & 0.88 & -0.07 & 0.28 & 0.55 & 0.85 & -0.07 & 0.27 & 0.54 \\
GDP deflator (\%) & -1.93 & -1.98 & -2.72 & -2.07 & 3.22 & 2.99 & 2.26 & 3.01 \\
CPI (\%) & & & & & & & & \\
$\quad$ Rural households & -2.67 & -2.48 & -3.10 & -2.69 & 2.32 & 2.43 & 1.82 & 2.26 \\
$\quad$ Urban households & -2.57 & -2.25 & -2.75 & -2.53 & 2.44 & 2.75 & 2.25 & 2.48 \\
$\quad$ Government & -0.70 & -1.27 & -1.28 & -0.95 & 4.59 & 3.92 & 3.93 & 4.31 \\
$\quad$ Regional average & -2.62 & -2.39 & -2.94 & -2.61 & 2.38 & 2.56 & 2.02 & 2.36 \\
Total utility (\%) & & & & & & & & \\
$\quad$ Rural households & 0.69 & 0.22 & 0.17 & 0.47 & 0.38 & 0.02 & 0.02 & 0.21 \\
$\quad$ Urban households & 1.84 & 1.23 & 1.42 & 1.64 & 1.30 & 0.76 & 1.11 & 1.16 \\
$\quad$ Government & 0.04 & -1.38 & -1.89 & -0.49 & 0.95 & -1.45 & -1.97 & 0.10 \\
$\quad$ Regional average & 1.11 & 0.40 & 0.58 & 0.85 & 0.91 & 0.11 & 0.36 & 0.62 \\
Equivalent variation (billion yuan) & & & & & & & \\
$\quad$ Rural households & 12.15 & 2.35 & 0.82 & 15.33 & 6.62 & 0.21 & 0.10 & 6.93 \\
$\quad$ Urban households & 38.50 & 8.33 & 10.24 & 57.07 & 27.11 & 5.16 & 8.01 & 40.28 \\
$\quad$ Government & 0.47 & -4.86 & -4.91 & -9.31 & 10.73 & -5.10 & -5.10 & 0.52 \\
$\quad$ Regional sum & 51.12 & 5.82 & 6.15 & 63.09 & 44.45 & 0.26 & 3.01 & 47.72 \\
Savings (nominal, \%) & & & & & & & & \\
$\quad$ Rural households & -1.47 & -2.01 & -2.62 & -1.03 & 3.49 & 2.89 & 2.21 & 4.13 \\
$\quad$ Urban households & -0.16 & -0.97 & -0.95 & -1.60 & 5.17 & 4.24 & 4.29 & 3.43 \\
$\quad$ Government & -3.43 & -2.68 & -3.16 & -1.33 & 1.56 & 2.37 & 1.87 & 3.82 \\
$\quad$ Regional average & -1.75 & -0.46 & -3.39 & -1.20 & 3.18 & 4.83 & 1.60 & 3.93 \\
Nominal exchange rate & & & & - & & & & 5.35 \\
Change in trade balance (billion yuan) & & -41.79 & & & & - \\
Terms of trade (\%) & & & & -0.46 & & & & \\
\hline
\end{tabular}

Source: Author's CERD simulation.
No change in trade balance

Eastern Central Western National Eastern Central Western National 
Table 6.9 Impact on output, exports and imports of WTO tariff cuts: with transfer payment

\begin{tabular}{|c|c|c|c|c|c|c|c|c|}
\hline \multirow[t]{2}{*}{ Sector ${ }^{a}$} & \multicolumn{4}{|c|}{ No control on trade balance } & \multicolumn{4}{|c|}{ No change in trade balance } \\
\hline & Eastern & Central & Western & National & Eastern & Central & Western & National \\
\hline \multicolumn{9}{|l|}{ Output } \\
\hline agri & - & -0.13 & -0.08 & -0.06 & -0.05 & -0.17 & -0.12 & -0.10 \\
\hline mine & -1.57 & 1.15 & 2.30 & -0.03 & -1.72 & 1.30 & 2.45 & -0.02 \\
\hline fprc & -4.19 & -1.72 & -5.37 & -3.59 & -4.32 & -1.80 & -5.54 & -3.71 \\
\hline lind & 7.78 & -0.07 & 2.58 & 5.90 & 7.87 & 0.09 & 3.15 & 6.03 \\
\hline chem & -1.95 & -1.01 & -0.90 & -1.62 & -1.98 & -0.96 & -0.84 & -1.61 \\
\hline motr & -17.73 & -14.99 & -12.13 & -16.38 & -17.89 & -14.71 & -11.69 & -16.37 \\
\hline mche & -3.75 & -1.05 & 0.01 & -2.74 & -3.81 & -0.85 & 0.20 & -2.71 \\
\hline elen & 5.84 & -0.97 & 2.69 & 5.04 & 5.87 & -0.88 & 2.97 & 5.10 \\
\hline cnst & 0.47 & 1.07 & 1.14 & 0.72 & 0.45 & 1.09 & 1.16 & 0.71 \\
\hline svce & 0.73 & -0.05 & 0.19 & 0.49 & 0.78 & -0.14 & 0.11 & 0.49 \\
\hline \multicolumn{9}{|l|}{ Exports } \\
\hline agri & 17.12 & 15.04 & 17.45 & 16.52 & 20.08 & 17.74 & 19.89 & 19.34 \\
\hline mine & 1.94 & 7.93 & 8.69 & 4.71 & 2.03 & 8.54 & 9.21 & 5.02 \\
\hline fprc & 9.60 & 9.59 & 6.84 & 9.17 & 10.94 & 10.99 & 7.76 & 10.46 \\
\hline lind & 21.89 & 8.61 & 18.68 & 20.13 & 22.54 & 9.65 & 21.19 & 20.90 \\
\hline chem & 5.01 & 5.81 & 6.31 & 5.20 & 5.27 & 6.32 & 6.85 & 5.52 \\
\hline motr & -10.08 & -5.71 & -0.05 & -7.87 & -10.15 & -5.06 & 0.73 & -7.71 \\
\hline mche & 1.34 & 5.61 & 6.98 & 2.61 & 1.46 & 6.23 & 7.51 & 2.85 \\
\hline elen & 15.15 & 5.44 & 12.09 & 14.52 & 15.44 & 5.91 & 12.81 & 14.84 \\
\hline cnst & 2.59 & 5.16 & 5.21 & 3.06 & 2.64 & 5.44 & 5.43 & 3.13 \\
\hline svce & 2.88 & 3.95 & 4.70 & 3.31 & 2.91 & 4.18 & 4.91 & 3.40 \\
\hline \multicolumn{9}{|l|}{ Imports } \\
\hline agri & 47.57 & 39.20 & 63.47 & 48.03 & 44.36 & 36.41 & 60.81 & 44.94 \\
\hline mine & -4.40 & -4.96 & -4.49 & -4.51 & -4.66 & -5.15 & -4.63 & -4.74 \\
\hline fprc & 155.46 & 173.65 & 158.35 & 158.35 & 151.16 & 169.31 & 155.16 & 154.28 \\
\hline lind & 22.54 & 11.11 & 5.13 & 19.04 & 21.96 & 9.95 & 4.14 & 18.34 \\
\hline chem & 22.45 & 19.33 & 20.84 & 21.77 & 22.19 & 18.91 & 20.45 & 21.46 \\
\hline motr & 110.72 & 107.98 & 88.18 & 106.10 & 109.91 & 106.75 & 87.48 & 105.20 \\
\hline mche & 12.58 & 13.47 & 12.62 & 12.72 & 12.34 & 13.12 & 12.37 & 12.46 \\
\hline elen & 6.63 & 3.92 & 4.34 & 5.93 & 6.31 & 3.45 & 3.98 & 5.58 \\
\hline cnst & -2.24 & -3.99 & -3.94 & -3.15 & -2.35 & -4.26 & -4.15 & -3.33 \\
\hline Svce & -2.37 & -4.75 & -4.97 & -3.47 & -2.50 & -5.18 & -5.35 & -3.73 \\
\hline
\end{tabular}

Note: ${ }^{a}$ sector code: agri: agriculture; mine: mining; fprc: food processing; lind: light industry; chem: chemicals; motr: motor vehicle and other transportation equipment; mche: machinery and equipment; elen: electronics and electric equipment; cnst: construction; svce: services.

Source: Author's CERD simulation. 
additional transfer payment to the inland regions, the total welfare gains decline from 63.69 and 48.04 billion yuan, respectively, to 63.09 and 47.72 billion yuan. The reduction in total welfare comes from the much sharper decline in government utility in the eastern region. It could be argued that government spending in the eastern region has higher returns, therefore a switch from eastern to inland regions causes efficiency losses. However, one cannot claim too much just based on this simulation, because the use of government spending is not specifically modelled and the transfer payment decision is not governed by an optimisation process.

\section{Conclusions}

Regional income disparity in China has been worsening since economic reforms began. Using a general equilibrium model of the Chinese economy with regional details (CERD), this chapter finds that this trend will be reinforced rather than eased by the WTO accession. The eastern coastal region will have much higher gains than the inland regions. The two inland regions will experience similar gains with the western region being only marginally better off than the central region. It is also found that the rural-urban inequality will worsen in all regions.

The simulation results are robust to whether the trade balance is fixed or not as the form of model closure. However, keeping the trade balance unchanged leads to smaller overall welfare gains and a wider regional income gap than when the trade balance is made endogenous.

Lowering the tariff cuts in agriculture reduces the total welfare gains, although it modifies the trend of worsening inequality between rural and urban households and between regions. Similarly, increasing transfer payments to the inland regions could marginally improve the regional and rural-urban inequality at the cost of a smaller overall welfare gain. However, allowing freer movement of factors and commodities across regions could improve the regional and rural-urban inequality and achieve higher total welfare gains.

Although most of the results derived by the analysis are consistent with other studies and people's perceptions, one should be cautious in accepting these results. First, this paper discusses only tariff cuts, rather than the whole framework of China's WTO commitments. The analysis could be extended to cover other issues, such as non tariff barriers, tariff rate 
quotas (TRQs) and domestic support. For example, the baseline tariff rates of the service sector are set at zero. Clearly, in reality this is not the case. The simulation using the closure of no control on trade balance shows that imports of crops increase by 84 to 88 per cent, which implies that the tariff rate quota for some crops may be binding. So the introduction of the TRQ in the model is necessary to obtain more realistic results.

Second, the CERD model is a national model, which suppresses international linkages and may omit some important information. For example, it predicts that China's apparel sector will increase following WTO accession. However, this result is very much dependent on whether other countries initiate the special textile safeguards. Therefore, it would be appropriate to link CERD with a global model to reflect these international relationships.

Third, the database and parameters need to be refined. For example, regional protection measures should be introduced. Also, the agricultural sectors could be disaggregated further as the present aggregation may hide significant impacts on some crops.

\section{Notes}

1 For example, see Li et al (1998), Wang (2000), lanchovichina and Martin (2001), Lloyd and Zhang (2001), Anderson, Huang and Ianchovichina (2002), Diao, Fan and Zhang (2002), Diao et al (2002), Francois and Spinanger (2002), and Yu and Frandsen (2002).

2 Although this is higher than the effective rate, it may represent the actual protection level if non- tariff barriers are taken into consideration. Moreover, the effective rate tends to underestimate the actual protection level as it is weighted by import volumes.

3 It should be noted that the treatment here implies no change in the employment (or unemployment) level embodied in the database.

4 It could be set at any level, but zero change is an obvious target.

5 It should be noted that China's crop imports account for only 1.3 per cent of total imports in the baseline, and that even after the 88 per cent increase crop imports are still small in absolute terms.

6 The problem of so-called san long (agriculture, farmers and rural development) has been a popular topic.

7 For example, the newly implemented reporting requirement for genetically modified food is interpreted as an important tool to protect China's traditional soybean growing areas in the northeast region against competition from the US. However, it should be pointed out that these practices have been learnt from other countries. China often complains that its exports face even stricter technical barriers. 


\section{References}

Anderson, K., Jikun Huang, and lanchovichina, E, 2002. 'Impact of China's WTO accession on rural-urban income inequality', Paper presented at the Australian Agricultural and Resource Economics Society Pre-Conference Workshop on WTO: Issues for Developing Countries, Canberra, 12 February 2002.

Chen, Chunlai, 2001. 'Foreign direct investment: prospects and policies', in OECD (ed.), China in the World Economy: domestic policy challenges, OECD, Paris:323-57.

Diao, Xinshen, Shenggen Fan and Xiaobo Zhang, 2002. How China's WTO accession affects rural economy in the less-developed regions: a multi-region, general equilibrium analysis, TMD Discussion Paper 87, International Food Policy Research Institute, Washington, DC.

Diao, Xinshen, Sherman Robinson, Agapi Somwaru, and Francis Tuan, 2002.

'Regional and national perspectives of China's integration into the WTO: a computable general equilibrium inquiry', Paper presented at the 5th conference on global economic analysis, Taipei, 5-7 June.

Fan, Mingtai and Yuxin Zheng. 2000. 'The impact of China's trade liberalisation for WTO accession: a computable general equilibrium analysis', Paper presented at the Third Annual Conference on Global Economic Analysis, 27-30 June, Monash University, Melbourne. Available at http://www.monash.edu.au/policy/conf/42fan.pdf.

,-- 2001. 'China's tariff reduction and WTO accession: a computable general equilibrium analysis', in P. Lloyd and Xiao-guang Zhang (eds), Models of the Chinese Economy, Edward Elgar, Cheltenham:211-35.

Francois, J.F., and Spinanger, D., 2002. 'Greater China's accession to the WTO: implication for international trade and for Hong Kong', Paper presented at the 5th conference on global economic analysis, Taipei, 5-7 June.

lanchovichina, E., and Martin, W., 2001. Trade liberalisation in China's accession to WTO', World Bank, Washington, DC (unpublished).

Jiang, Tingsong. 2002. 'WTO accession and regional incomes', in Garnaut, R. and Ligang Song (eds), China 2002: WTO Entry and World Recession, Asia Pacific Press, Canberra:45-62. 
Kehoe, T.J., 2002. 'An evaluation of the performance of applied general equilibrium models of the impact of NAFTA', Keynote speech at the fifth conference on global economic analysis, Taipei, 5-7 June.

Lardy, N.R., 2002. Integrating China into the Global Economy, Brookings Institution, Washington, DC.

$\mathrm{Li}$, Shantong, Zhi Wang, Fan Zhai, and Lin Xu, 1998. The global and domestic impact of China joining the World Trade Organisation, Research Report, Washington Centre for China Studies and Development Research Center, the State Council, People's Republic of China.

Lloyd, Peter, and Xiao-guang Zhang, eds., 2001. Models of the Chinese Economy, Edward Elgar, Cheltenham.

National Bureau of Statistics of China, 2001. Statistical Yearbook of China, China Statistics Press, Beijing.

Sun, Haishun. 2000. 'Economic growth and regional disparity in China', Regional Development Studies, 6:43-66.

Sun, Haishun and Ashor Parikh, 2001. 'Exports, inward foreign direct investment (FDI) and regional economic growth in China', Regional Studies, 35(3):187-96.

Wang, Zhi, 2000. The economic impact of China's WTO accession on the world economy, Economic Research Service, US Department of Agriculture, Washington, DC (unpublished).

World Trade Organization, 2001. China's WTO Accession Protocol, World Trade Organization, Geneva. Available online at http://www.wto.org/english/ thewto_e/acc_e/completeacc_e.htm

Wu, Yanrui, 1999. Income disparity and convergence in China's regional economies, Discussion Paper 99-15, Department of Economics, University of Western Australia. Available at http://www.econs.ecel.uwa.edu.au/ economics/dpapers/DP1999/9.15.pdf, last accessed on 2 January 2002.

Yang, Yongzheng and Yiping Huang, 1997. The impact of trade liberalisation on income distribution in China, Economics Division Working Papers, China Economy 97/1, Research School of Pacific and Asian Studies, The Australian National University, Canberra.

Yu, Wusheng, and Frandsen, Søren E., 2002. 'China's WTO commitments in agriculture: does the impact depend on OECD agricultural policies', Paper presented at the 5th conference on global economic analysis, Taipei, 5-7 June. 


\section{Appendix}

\section{Table A6.1 Sector classification in the CERD model}

Sectors in the CERD model

Agriculture

01 Crops

02 Forestry

03 Livestock and livestock products

Industry and construction

06 Coal mining and processing

07 Crude petroleum and natural gas products

08 Metal ore mining

09 Non metal mineral mining

10 Manufacture of food products and tobacco processing

11 Textile goods

12 Wearing apparel, leather, furs, down and related products

13 Sawmills and furniture

14 Paper and products, printing and record medium reproduction

15 Petroleum processing and coking

16 Chemicals

17 Non metal mineral products

18 Metals smelting and pressing

19 Metal products

Services

32 Transport and warehousing

33 Post and telecommunication

34 Wholesale and retail trade

35 Eating and drinking places

36 Passenger transport

37 Finance and issurance

38 Real estate

39 Social services
Code Sectors in the CERD model

Code

crop $\quad 04$ Fishery

fish

frst

05 Other agricultural products

otha coal 20 Machinery and equipment mach

petr 21 Transport equipment trep

22 Electric equipment and machinery eltc

mtom 23 Electronic and telecommunication eltn

nmtm equipment

fdtp 24 Instruments, meters, cultural and inst office machinery

txtl 25 Maintenance and repair of machine main

aprl

furn

papr

ptpc

chem

nmmp

$\mathrm{mtsp}$

mtlp

tran

ptlc

trad

cate

past

fina

rest

sser
40 Health services, sports and social heth welfare

41 Education, culture and arts, radio, educ film and television

42 Scientific research scir

43 General technical services teks

44 Public administration and other padm sectors 
Table A6.2 Baseline and WTO commitment tariff rates

\begin{tabular}{lccclccc}
\hline Sector & $\begin{array}{c}\text { Baseline } \\
\text { rate }\end{array}$ & $\begin{array}{c}\text { WTO } \\
\text { rate }\end{array}$ & $\begin{array}{c}\text { Change } \\
\text { (per cent) }\end{array}$ & Sector & $\begin{array}{c}\text { Baseline } \\
\text { rate }\end{array}$ & $\begin{array}{c}\text { WTO } \\
\text { rate }\end{array}$ & $\begin{array}{c}\text { Change } \\
\text { (per cent) }\end{array}$ \\
crop & 27.70 & 16.88 & -39.06 & eltn & 13.54 & 10.00 & -26.15 \\
frst & 2.65 & 2.00 & -24.41 & inst & 13.54 & 10.00 & -26.15 \\
live & 17.63 & 15.00 & -14.93 & main & - & - & - \\
fish & 16.59 & 15.00 & -9.59 & omnp & 26.25 & 15.00 & -42.86 \\
otha & 59.01 & 17.00 & -71.19 & scrp & - & - & - \\
coal & 4.97 & 1.26 & -74.65 & powr & -0.01 & - & - \\
petr & - & - & -46.24 & gasp & - & - & - \\
mtom & - & - & - & watr & - & - & - \\
nmtm & 0.44 & - & - & cons & - & - & - \\
fdtp & 52.90 & 20.00 & -62.20 & tran & - & - & - \\
txtl & 33.49 & 20.00 & -40.28 & ptlc & - & - & - \\
aprl & 25.55 & 25.00 & -2.15 & trad & - & - & - \\
furn & 13.81 & 10.00 & -27.60 & cate & - & - & - \\
papr & 13.19 & 10.00 & -24.17 & past & - & - & - \\
ptpc & 9.09 & 7.00 & -23.01 & fina & - & - & - \\
chem & 15.41 & 5.00 & -67.55 & rest & - & - & - \\
nmmp & 22.12 & 20.00 & -9.60 & sser & - & - & - \\
mtsp & 9.85 & 7.00 & -28.91 & heth & - & - & - \\
mtlp & 15.37 & 7.00 & -54.46 & educ & - & - & - \\
mach & 15.62 & 11.00 & -29.57 & scir & - & - & - \\
treq & 23.26 & 10.00 & -57.01 & teks & - & - & - \\
eltc & 13.54 & 10.00 & -26.15 & padm & - & - & - \\
& & & & & & & \\
\hline & & & & & & & \\
\hline
\end{tabular}


Table A6.3 Percentage change in output - full WTO tariff cuts

\begin{tabular}{|c|c|c|c|c|c|c|c|c|}
\hline \multirow[b]{2}{*}{ Sector } & \multicolumn{4}{|c|}{ No control on trade balance } & \multicolumn{4}{|c|}{ No change in trade balance } \\
\hline & Eastern & Central & Western & National & Eastern & Central & Western & Nationa \\
\hline crop & -1.89 & -0.57 & -0.81 & -1.22 & -1.90 & -0.58 & -0.78 & -1.22 \\
\hline frst & 1.84 & 1.34 & 1.52 & 1.60 & 2.20 & 1.54 & 1.66 & 1.86 \\
\hline live & 2.96 & 0.35 & 1.01 & 1.63 & 2.88 & 0.27 & 0.88 & 1.54 \\
\hline fish & -0.09 & 0.29 & 0.28 & - & -0.34 & 0.11 & -0.07 & -0.23 \\
\hline otha & 1.18 & -0.04 & -0.09 & 0.61 & 1.07 & -0.25 & -0.12 & 0.49 \\
\hline coal & -1.94 & 0.54 & 0.51 & -0.44 & -2.21 & 0.61 & 0.53 & -0.52 \\
\hline petr & -1.05 & 7.74 & 4.36 & 0.89 & -1.10 & 8.47 & 4.59 & 0.96 \\
\hline mtom & -3.99 & 1.08 & 3.30 & -0.92 & -4.11 & 1.39 & 3.62 & -0.82 \\
\hline $\mathrm{nmtm}$ & -0.75 & 1.46 & 1.52 & 0.39 & -0.90 & 1.57 & 1.54 & 0.36 \\
\hline fdtp & -4.20 & -1.71 & -5.35 & -3.59 & -4.33 & -1.80 & -5.53 & -3.71 \\
\hline txtl & 8.11 & -1.20 & 7.46 & 6.34 & 8.40 & -0.66 & 9.23 & 6.79 \\
\hline aprl & 17.14 & 0.27 & 1.49 & 14.42 & 17.29 & 0.18 & 1.32 & 14.53 \\
\hline furn & 0.51 & 1.36 & 1.16 & 0.81 & 0.35 & 1.35 & 1.05 & 0.69 \\
\hline papr & 1.92 & -0.11 & -0.80 & 1.32 & 1.96 & -0.11 & -0.75 & 1.35 \\
\hline ptpc & -1.30 & 0.66 & 2.34 & -0.41 & -1.41 & 0.80 & 2.48 & -0.43 \\
\hline chem & -3.05 & -3.89 & -3.07 & -3.21 & -3.01 & -3.85 & -2.96 & -3.16 \\
\hline $\mathrm{nmmp}$ & -0.16 & 1.40 & 1.27 & 0.52 & -0.29 & 1.45 & 1.24 & 0.46 \\
\hline mtsp & -4.89 & -0.51 & 1.29 & -2.73 & -5.02 & -0.24 & 1.51 & -2.71 \\
\hline mtlp & -2.82 & -1.95 & -2.63 & -2.66 & -2.83 & -1.86 & -2.57 & -2.65 \\
\hline mach & -3.98 & -1.10 & -0.78 & -3.15 & -4.00 & -0.91 & -0.54 & -3.10 \\
\hline treq & -17.88 & -14.87 & -11.54 & -16.37 & -18.05 & -14.58 & -11.10 & -16.36 \\
\hline eltc & 0.52 & 0.23 & 0.73 & 0.50 & 0.37 & 0.31 & 0.84 & 0.39 \\
\hline eltn & 11.04 & -2.99 & 5.62 & 9.93 & 11.22 & -2.92 & 6.04 & 10.13 \\
\hline inst & 5.24 & -2.38 & -0.93 & 3.95 & 5.45 & -2.15 & -0.63 & 4.17 \\
\hline main & -0.46 & -0.13 & 0.86 & -0.16 & -0.51 & -0.10 & 0.87 & -0.18 \\
\hline omnp & -0.87 & 0.94 & -0.06 & -0.30 & -1.11 & 1.08 & -0.02 & -0.41 \\
\hline scrp & -3.22 & -1.08 & 4.86 & -1.88 & -3.34 & -1.01 & 5.12 & -1.91 \\
\hline powr & -0.45 & 0.10 & 0.40 & -0.19 & -0.62 & 0.13 & 0.46 & -0.28 \\
\hline gasp & 1.16 & 1.25 & 0.89 & 1.15 & 0.48 & 0.38 & 0.75 & 0.49 \\
\hline watr & 0.54 & 0.03 & 0.24 & 0.38 & 0.36 & -0.16 & 0.09 & 0.20 \\
\hline cons & 0.46 & 1.09 & 1.18 & 0.72 & 0.43 & 1.11 & 1.20 & 0.72 \\
\hline $\operatorname{tran}$ & 0.06 & 0.41 & 0.99 & 0.25 & -0.05 & 0.48 & 1.05 & 0.20 \\
\hline ptlc & 1.19 & 0.30 & 0.52 & 0.93 & 0.98 & 0.08 & 0.32 & 0.72 \\
\hline trad & 0.59 & 0.08 & 1.14 & 0.56 & 0.40 & 0.07 & 1.14 & 0.44 \\
\hline cate & 1.71 & 0.63 & 0.67 & 1.32 & 1.46 & 0.34 & 0.35 & 1.05 \\
\hline past & 0.76 & 0.47 & 0.97 & 0.71 & 0.66 & 0.33 & 0.91 & 0.60 \\
\hline fina & 0.17 & 0.06 & 0.49 & 0.20 & -0.09 & 0.08 & 0.58 & 0.04 \\
\hline rest & 1.17 & 0.45 & 0.69 & 0.98 & 0.79 & -0.03 & 0.37 & 0.59 \\
\hline sser & 1.50 & 0.30 & 0.31 & 1.16 & 1.59 & 0.20 & 0.21 & 1.19 \\
\hline heth & 2.05 & -0.80 & -0.66 & 0.84 & 2.47 & -1.02 & -1.02 & 0.96 \\
\hline educ & 1.49 & -0.79 & -0.72 & 0.61 & 1.95 & -1.11 & -1.06 & 0.75 \\
\hline scir & 0.55 & -1.81 & -1.89 & -0.26 & 1.18 & -1.86 & -1.92 & 0.14 \\
\hline teks & 0.68 & -1.44 & -1.67 & -0.14 & 1.63 & -1.51 & -1.73 & 0.44 \\
\hline padm & 1.87 & -1.97 & -2.53 & 0.16 & 3.33 & -2.04 & -2.60 & 0.98 \\
\hline
\end{tabular}


స్ 耍

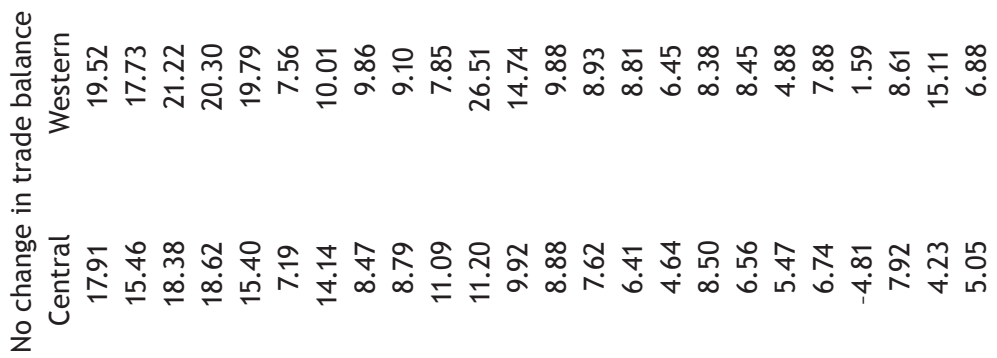

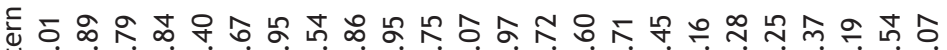
岕 穴

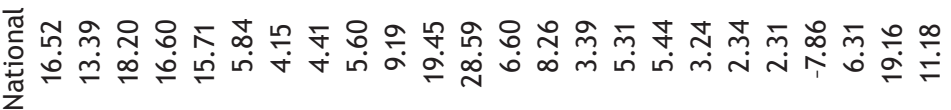
$\stackrel{\circ}{3} \quad \stackrel{0}{2}$

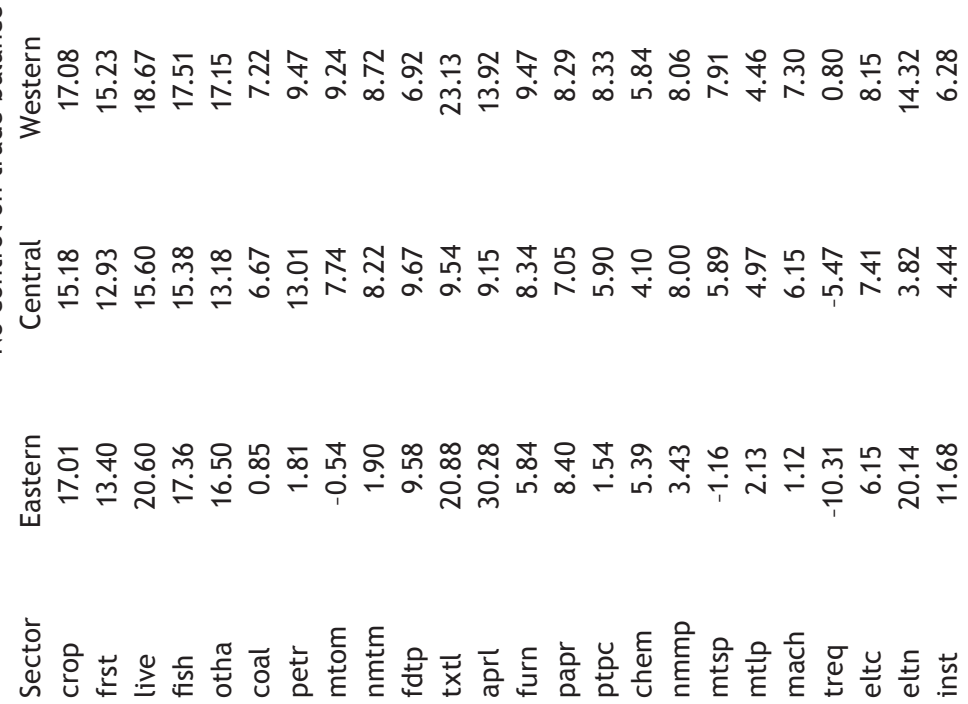


Agriculture and Food Security in China

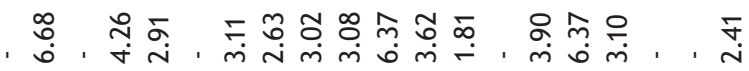

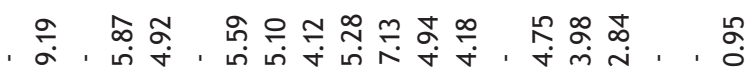

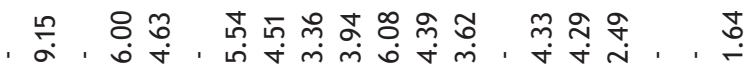

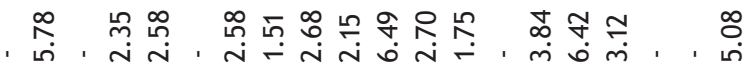

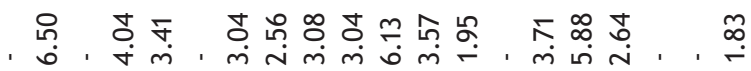

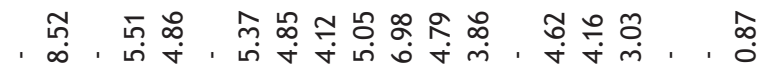

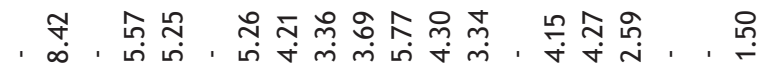

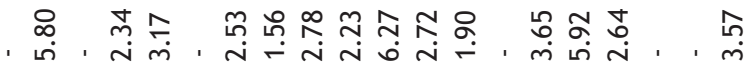

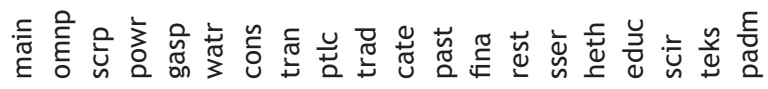




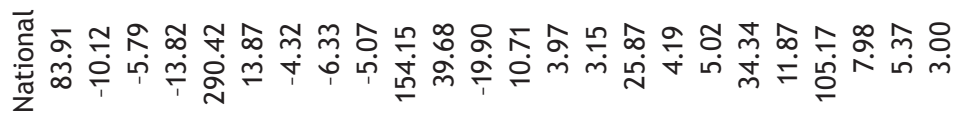

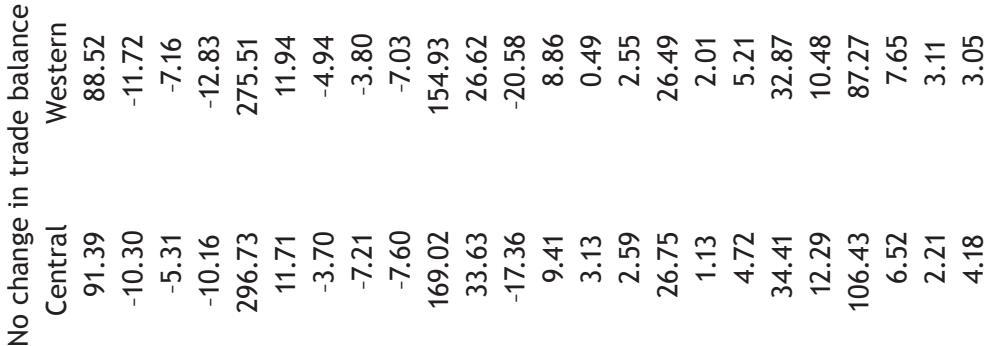

๘ 岕市

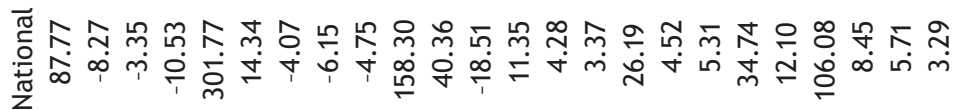
우

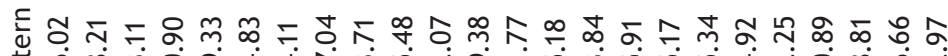

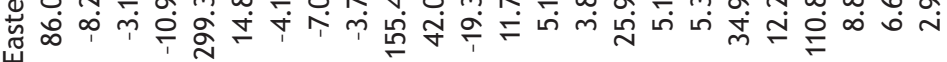


Agriculture and Food Security in China

舟

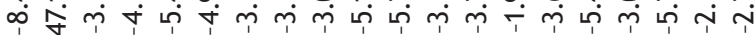

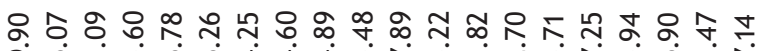
वे

๖ ำ 눈

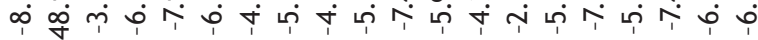

ச ले

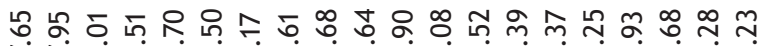

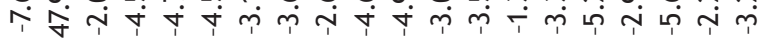

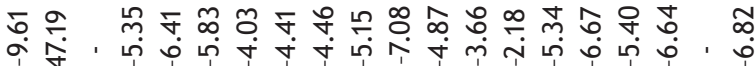

กิ ธ क

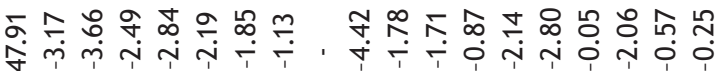

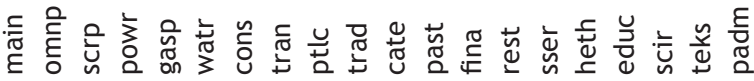




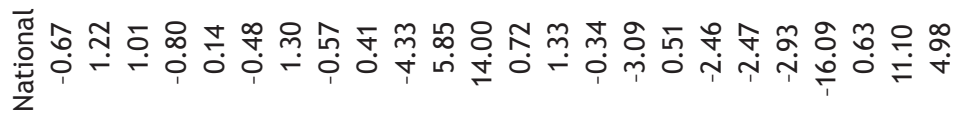

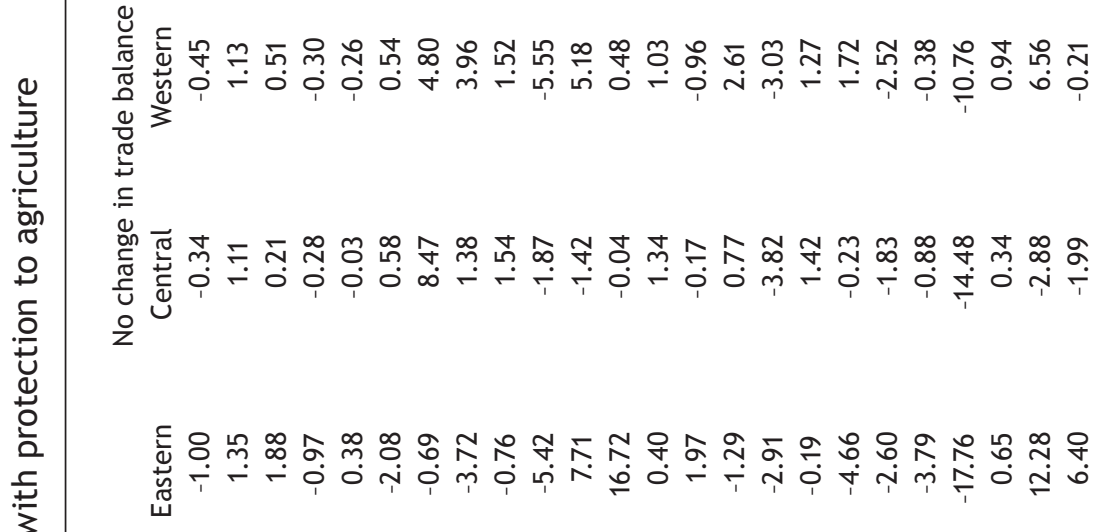

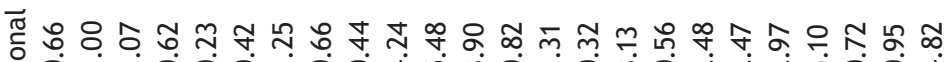
吾

$\stackrel{\circ}{\lessgtr}$

$\stackrel{+}{\stackrel{1}{3}}$

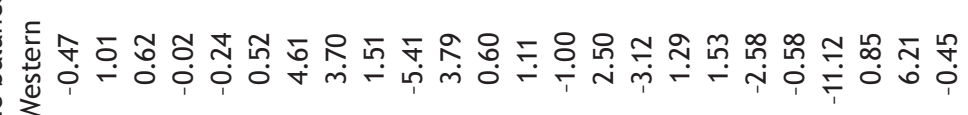

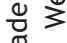

$\subseteq$ 車

ถัก ธิ

$\frac{\frac{1}{\sigma}}{\cup}$

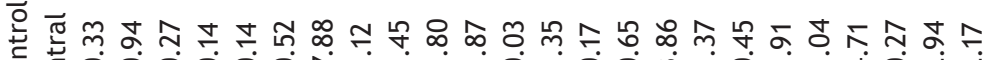

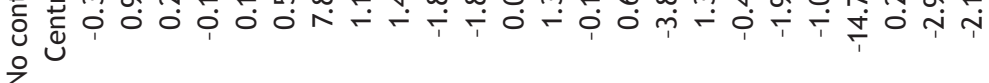
ণ্ণ

๘ 崫

䎹

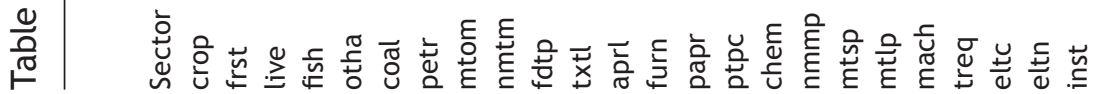


Agriculture and Food Security in China

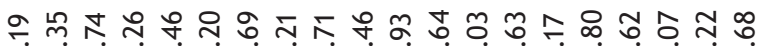

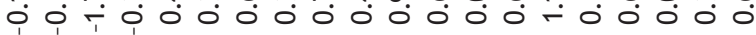

$\infty$ m రำ

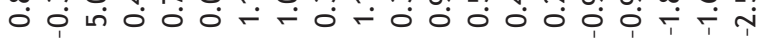

Ғ

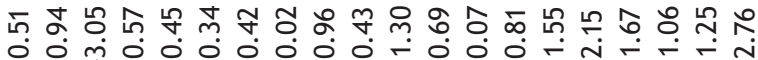

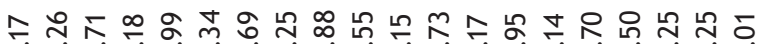
宁

ఏ m

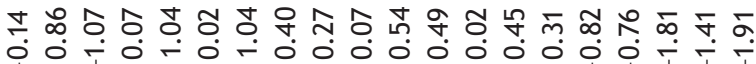

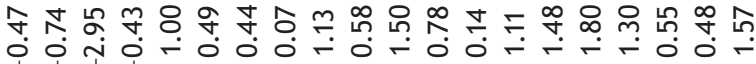

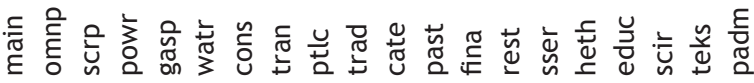




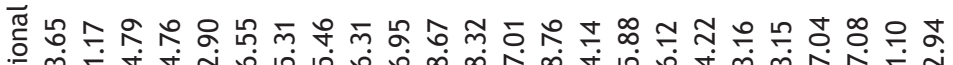

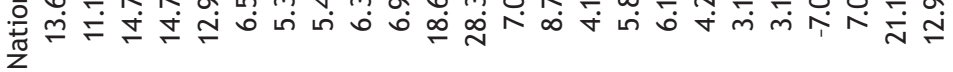

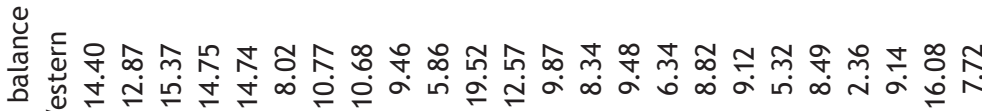
$\frac{1}{3} 3$

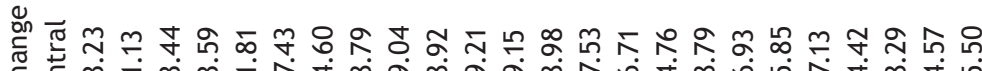

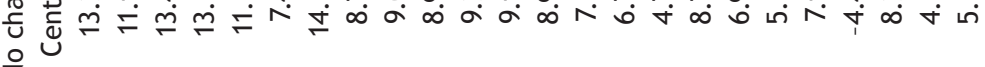
으

도 ๙ 岀

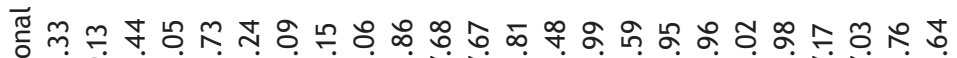
宾兵

0

논 $\stackrel{1}{2}$ $\frac{\pi}{3}$

.$\subseteq$

ถัก ว

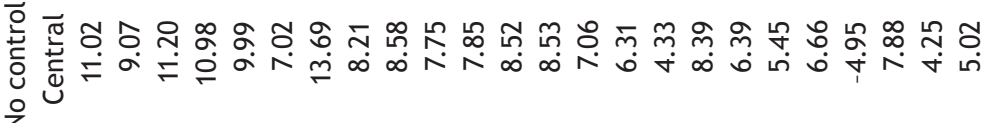
은

둰 ㅇํำ

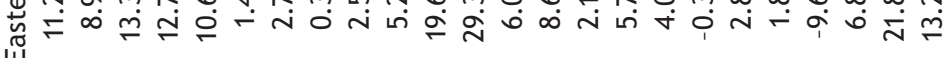

กั

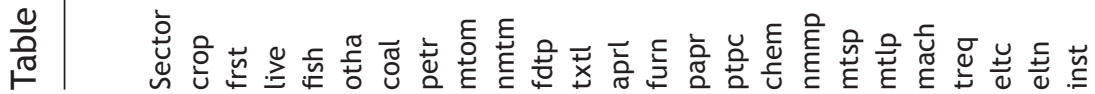


Agriculture and Food Security in China

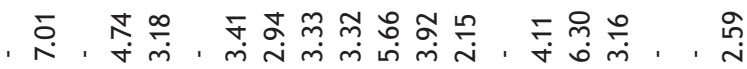

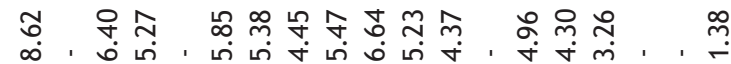

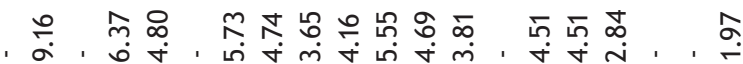

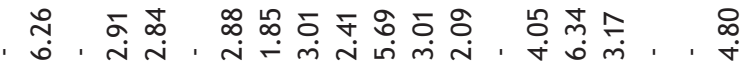

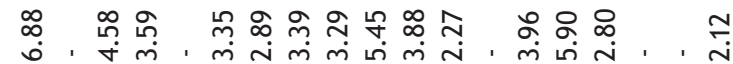

,

, ஸீ,

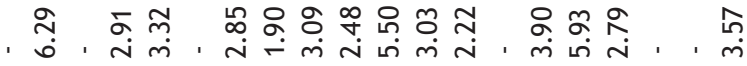

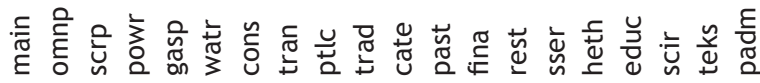




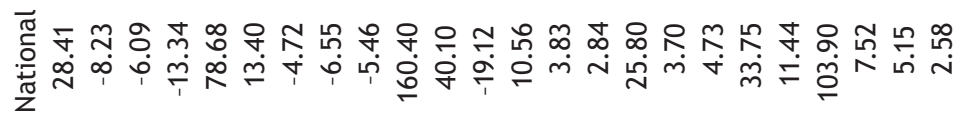

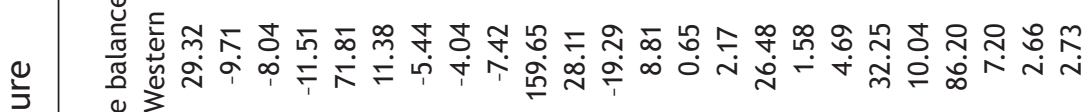

至 $\quad \frac{\pi}{2}$

돈

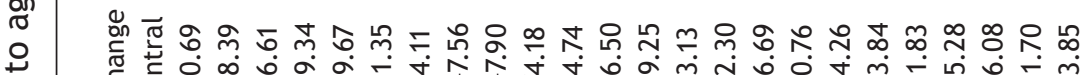

든 $\quad$ 는

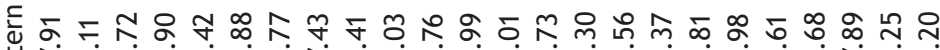
岕

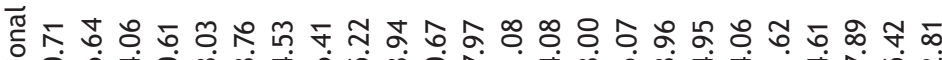
范

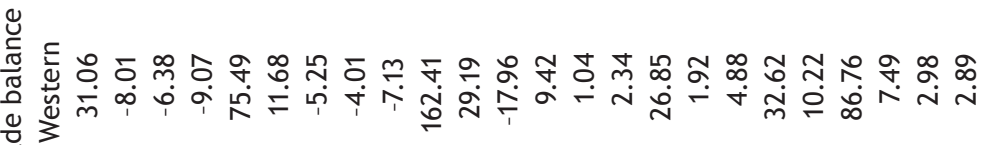
乔

ำ 岕 
Agriculture and Food Security in China

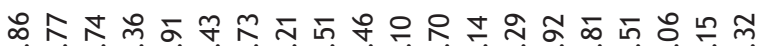

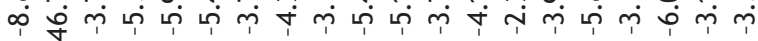

๓ 官的

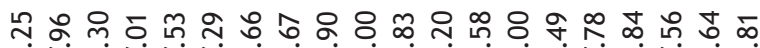

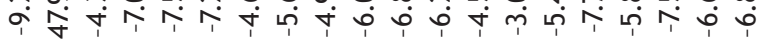

ำก

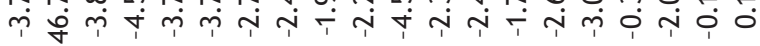

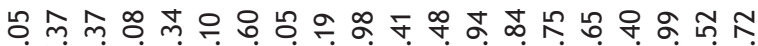
め

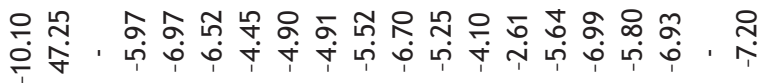

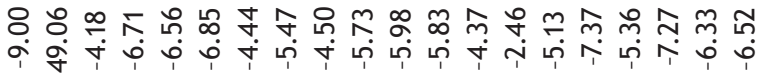

సิ ํํำ -

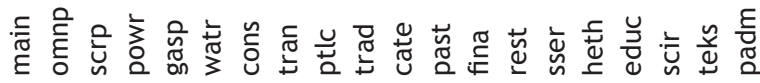




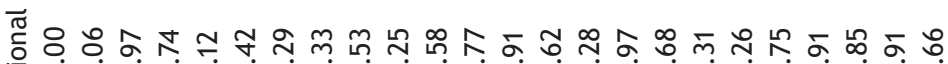
元

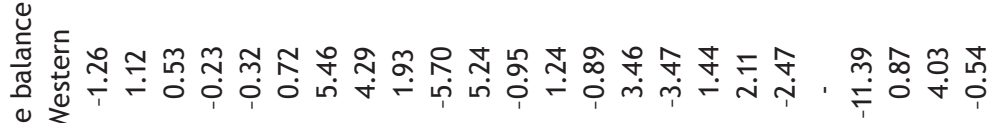
कृ

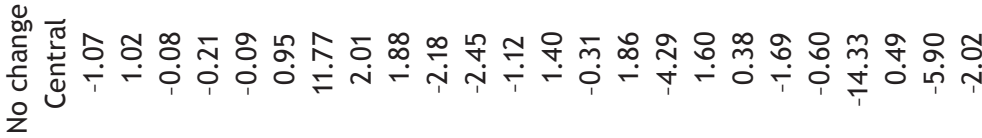

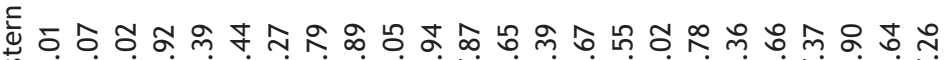
嵌

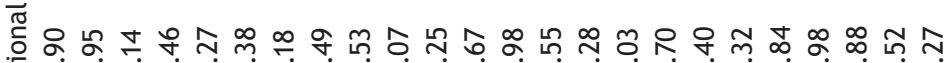
花

ه

离 (1) 3

$\subseteq$ 芒

ชิ ธิ

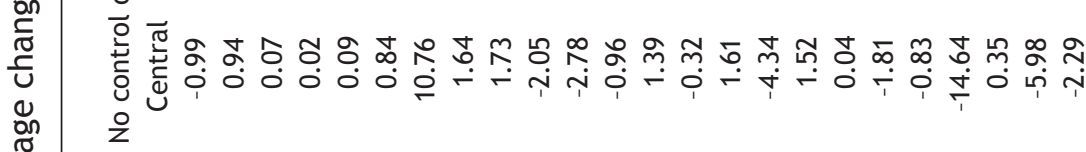

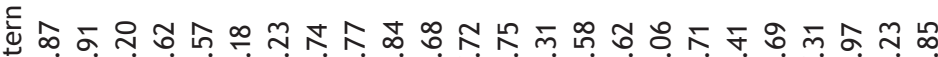
岕

๕.

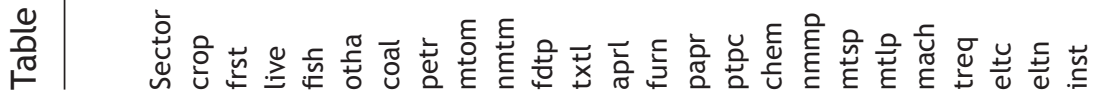


Agriculture and Food Security in China

Ғ

華 革

๓

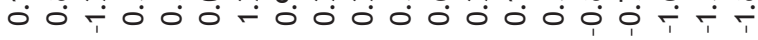

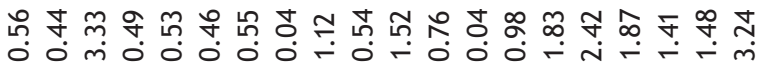

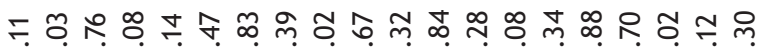

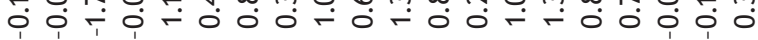

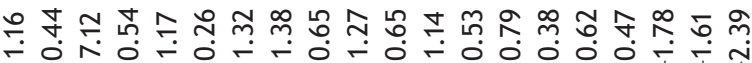

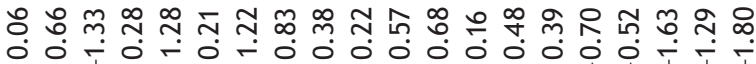

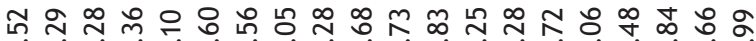

○ं

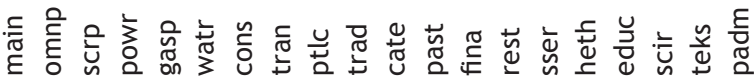




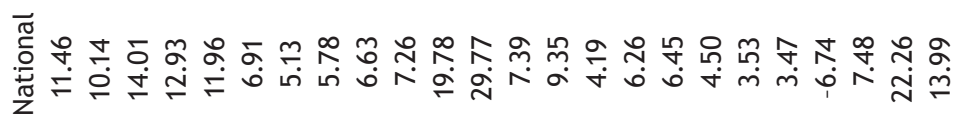

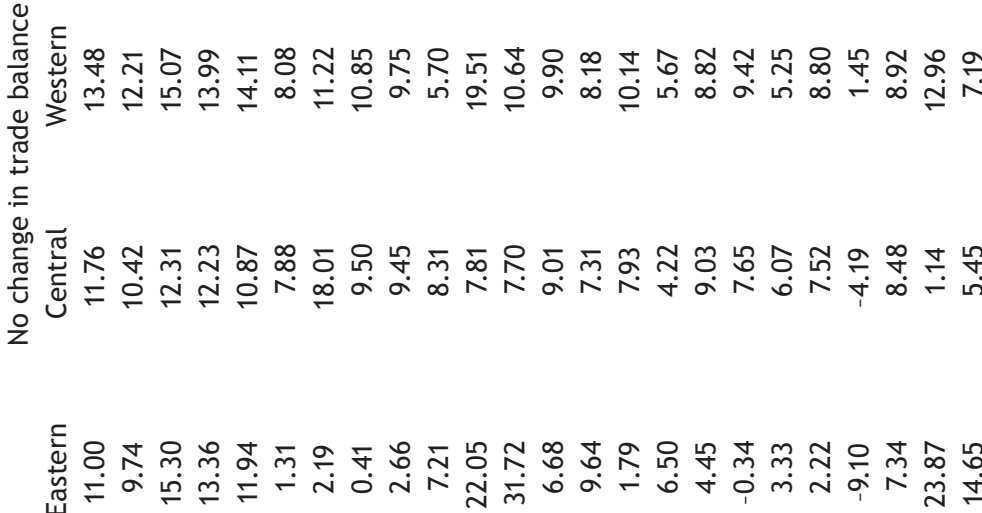

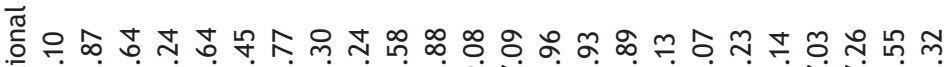

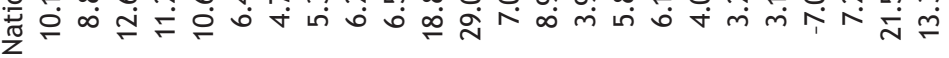

(1)

空

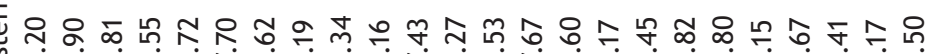


๓ัต

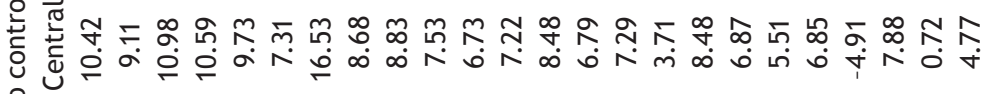
은

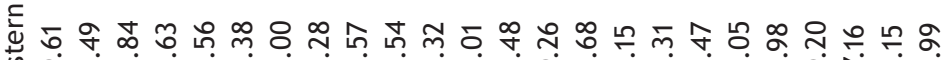

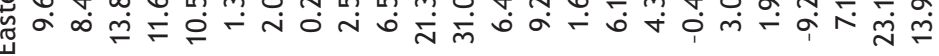

운

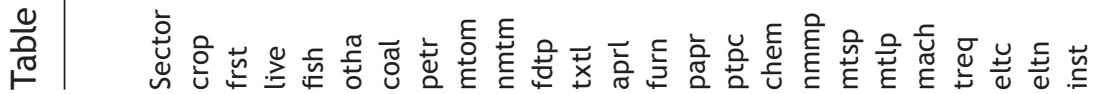


Agriculture and Food Security in China

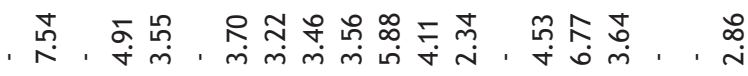

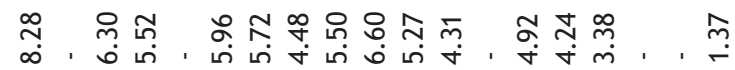

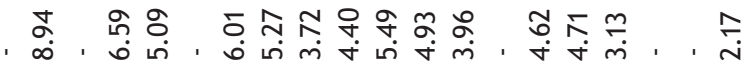

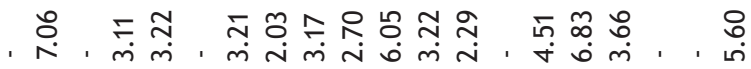

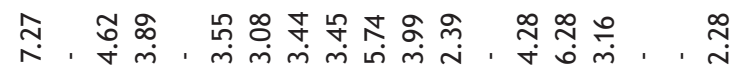

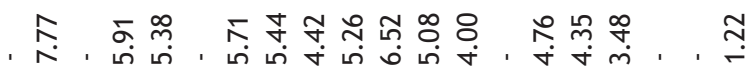

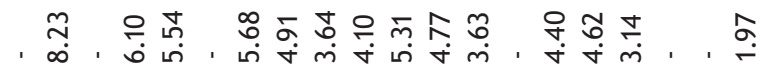

, 朵,

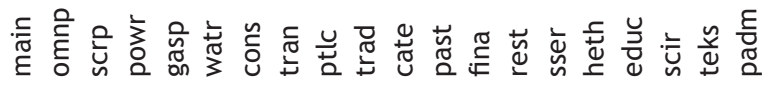




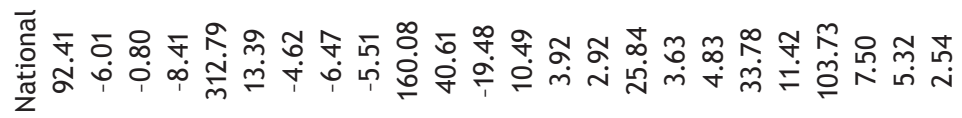

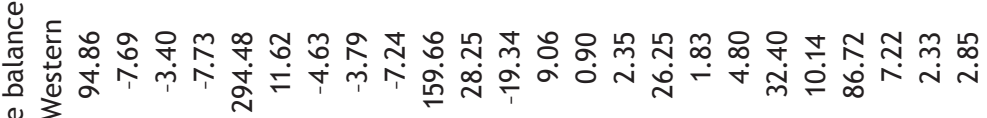
岸

인

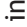

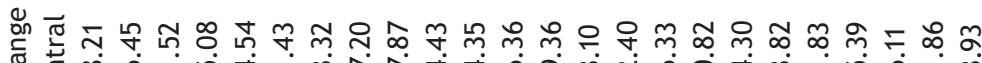
宅过 은

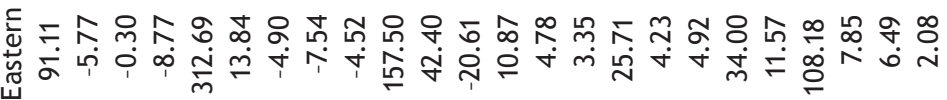

స్ 旁

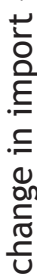

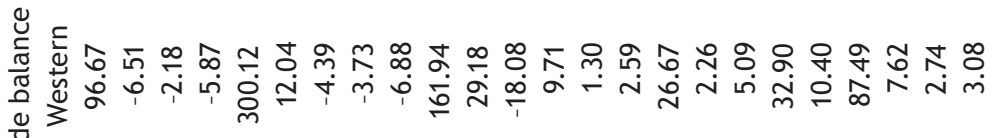
\% ธ

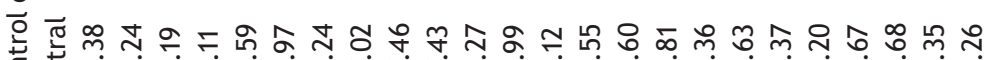

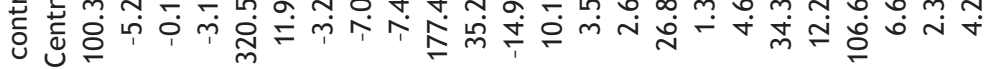
운

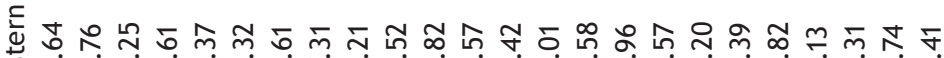

F 岀

䢓

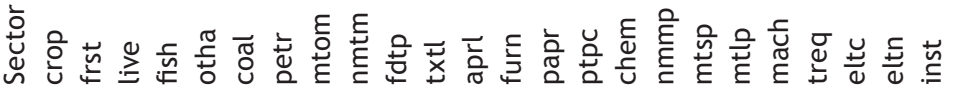


Agriculture and Food Security in China

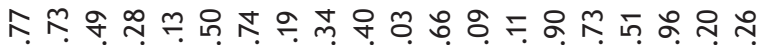

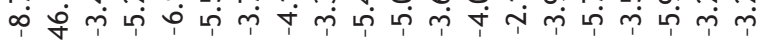

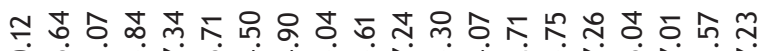

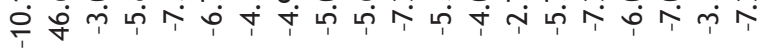

กั

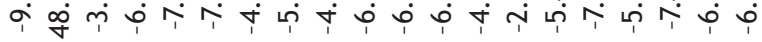

ம

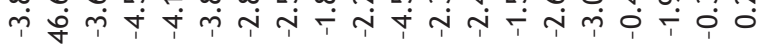

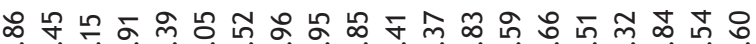

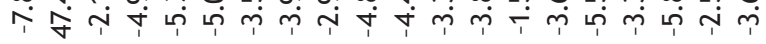

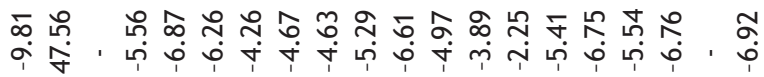

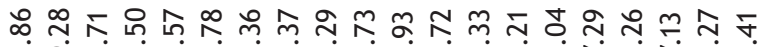
㝖

๓

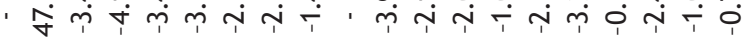

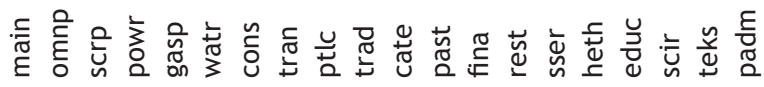




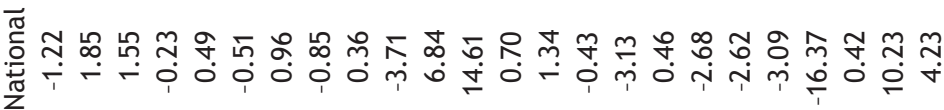

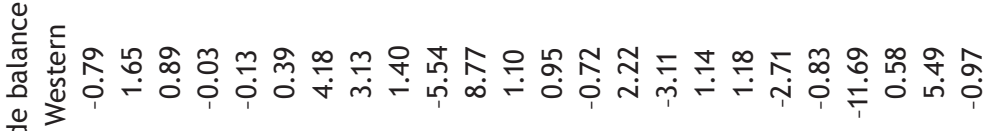
कृ

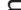

品

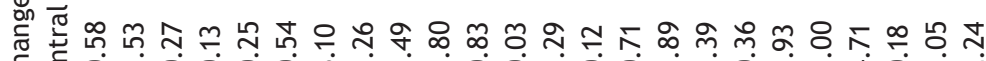
岂

我

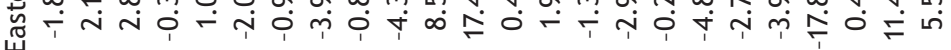

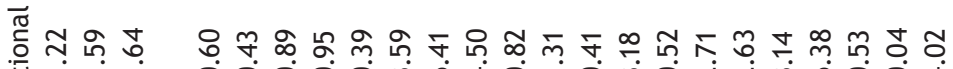
華

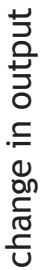

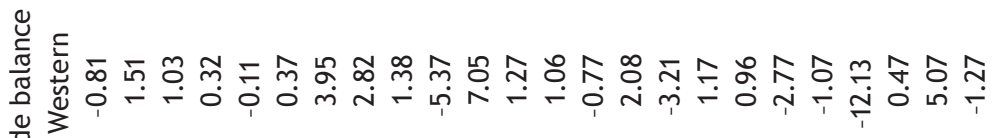
\& 3

ه ธิ

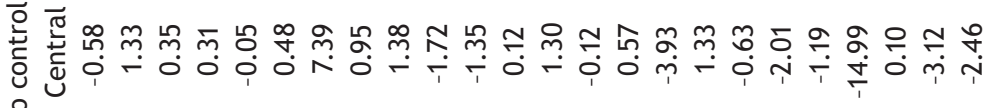
苋

$\frac{1}{\square}$

N

동

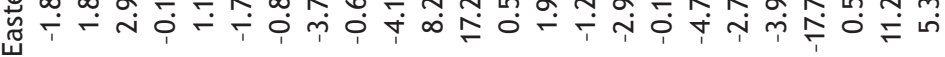

电

$\frac{0}{\frac{0}{\sigma}}$

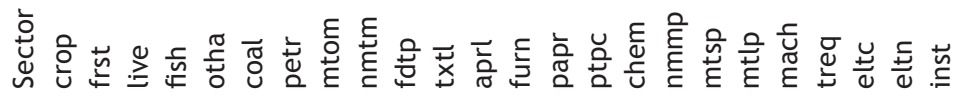


Agriculture and Food Security in China

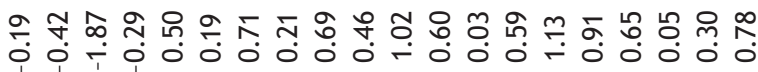

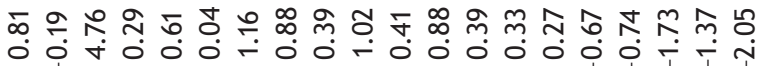
둥 \&

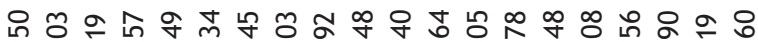
○े

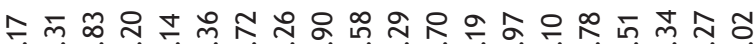
宁

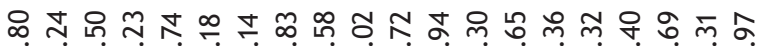
○े

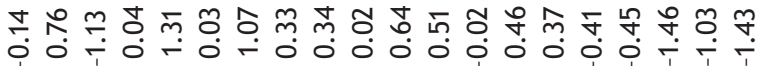

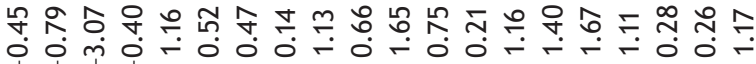

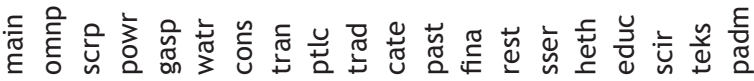




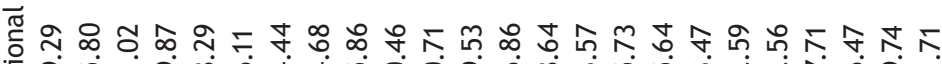

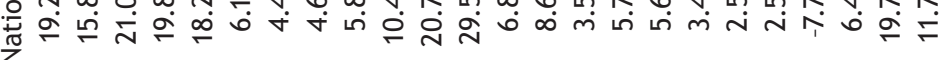

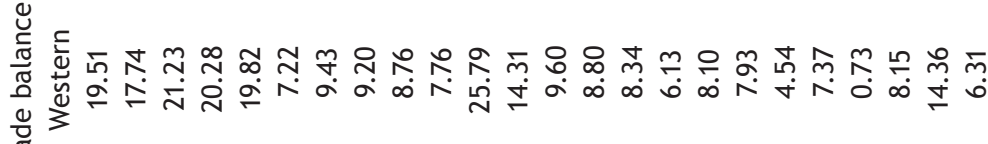
ㅁํㄴ .$\subseteq$

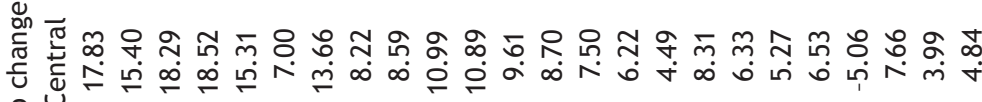
운

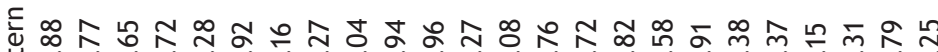
范

ธี 䒕

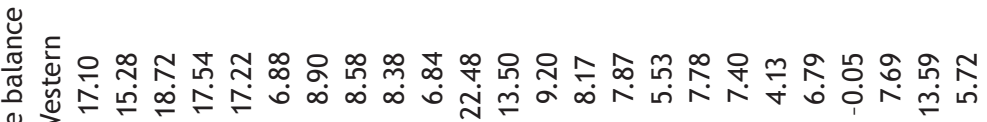
$\frac{1}{2} \sum^{\infty}$ \% ㄷํㅇ

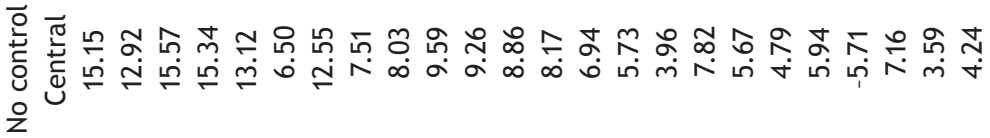

돓 m.

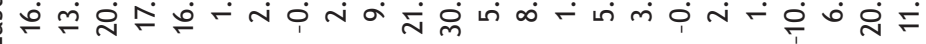

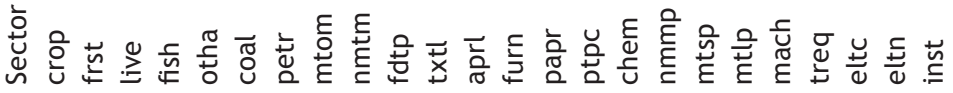


Agriculture and Food Security in China

, 审, 守

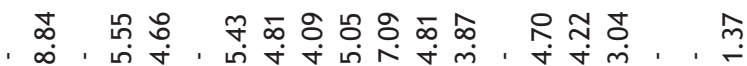

, $\begin{aligned} & \infty \\ & \infty\end{aligned}$,

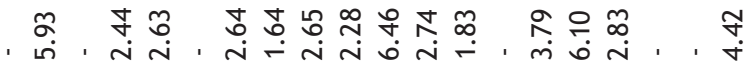

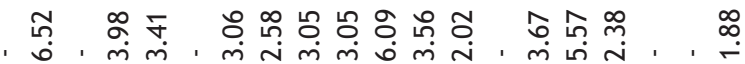

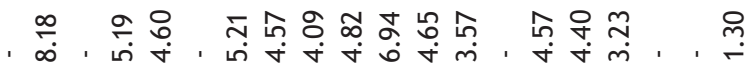

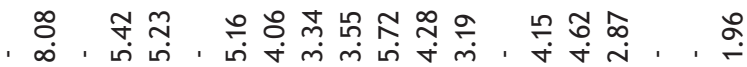

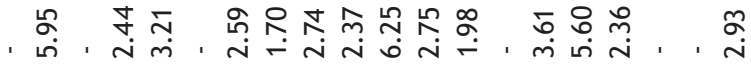

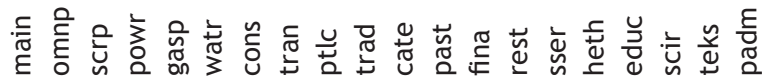




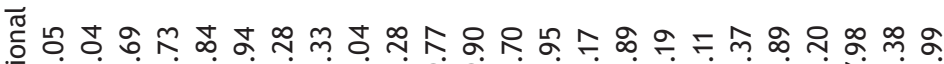
茂 市 우

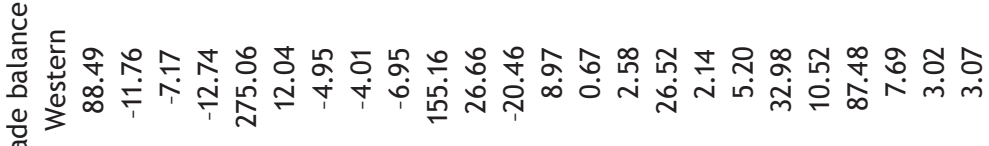

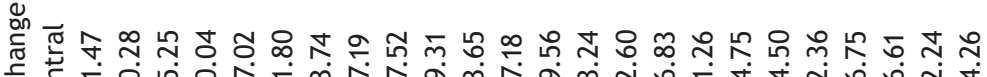

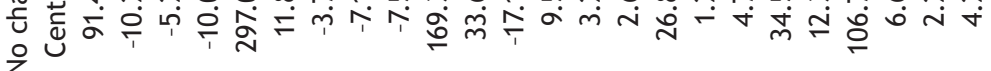
z

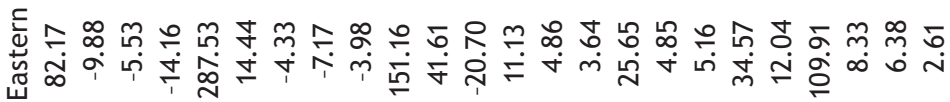

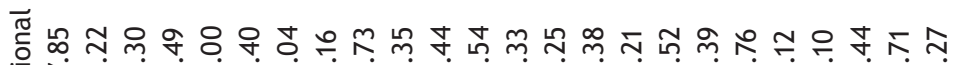

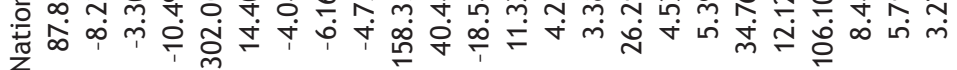

tั้

트

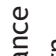

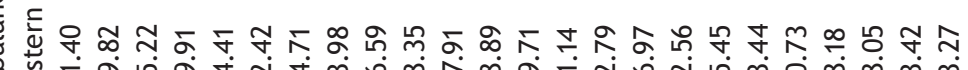
峎示

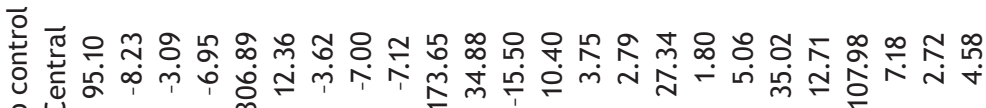

눤 岁 $\infty$

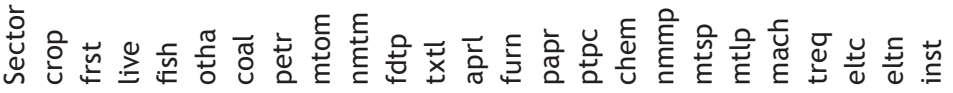


Agriculture and Food Security in China

ஸेサ

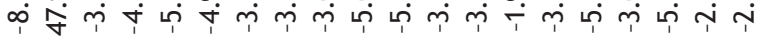

구ㅇㅠㅠ वे

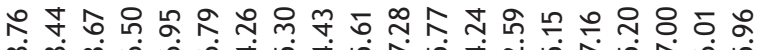
車

เก

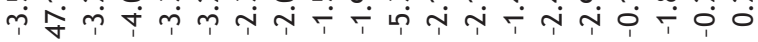

ํํ

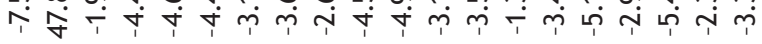

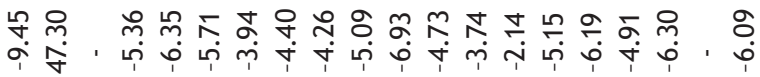

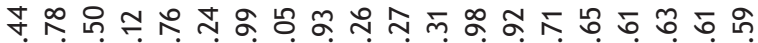
守

金

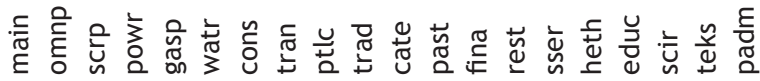

